\title{
Ideals in big Lipschitz algebras of analytic functions
}

\author{
by \\ Thomas Vils Pedersen (Frederiksberg)
}

\begin{abstract}
For $0<\gamma \leq 1$, let $\Lambda_{\gamma}^{+}$be the big Lipschitz algebra of functions analytic on the open unit disc $\mathbb{D}$ which satisfy a Lipschitz condition of order $\gamma$ on $\overline{\mathbb{D}}$. For a closed set $E$ on the unit circle $\mathbb{T}$ and an inner function $Q$, let $J_{\gamma}(E, Q)$ be the closed ideal in $\Lambda_{\gamma}^{+}$ consisting of those functions $f \in \Lambda_{\gamma}^{+}$for which

(i) $f=0$ on $E$,

(ii) $|f(z)-f(w)|=o\left(|z-w|^{\gamma}\right)$ as $d(z, E), d(w, E) \rightarrow 0$,

(iii) $f / Q \in \Lambda_{\gamma}^{+}$.

Also, for a closed ideal $I$ in $\Lambda_{\gamma}^{+}$, let $E_{I}=\{z \in \mathbb{T}: f(z)=0$ for every $f \in I\}$ and let $Q_{I}$ be the greatest common divisor of the inner parts of non-zero functions in $I$. Our main conjecture about the ideal structure in $\Lambda_{\gamma}^{+}$is that $J_{\gamma}\left(E_{I}, Q_{I}\right) \subseteq I$ for every closed ideal $I$ in $\Lambda_{\gamma}^{+}$. We confirm the conjecture for closed ideals $I$ in $\Lambda_{\gamma}^{+}$for which $E_{I}$ is countable and obtain partial results in the case where $Q_{I}=1$. Moreover, we show that every $\mathrm{wk}^{*}$ closed ideal in $\Lambda_{\gamma}^{+}$is of the form $\left\{f \in \Lambda_{\gamma}^{+}: f=0\right.$ on $E$ and $\left.f / Q \in \Lambda_{\gamma}^{+}\right\}$for some closed set $E \subseteq \mathbb{T}$ and some inner function $Q$.
\end{abstract}

1. Introduction. Throughout this paper, we let $0<\gamma \leq 1$ unless otherwise stated and denote all constants by $C$. Let $\Lambda_{\gamma}$ be the big Lipschitz algebra of functions $f$ on the unit circle $\mathbb{T}$ for which

$$
|f(z)-f(w)| \leq C|z-w|^{\gamma}
$$

for $z, w \in \mathbb{T}$. Equipped with the norm

$$
\|f\|_{\Lambda_{\gamma}}=\|f\|_{\infty}+\sup \left\{\frac{|f(z)-f(w)|}{|z-w|^{\gamma}}: z, w \in \mathbb{T}, z \neq w\right\} \quad\left(f \in \Lambda_{\gamma}\right),
$$

it is well known to be a Banach algebra. We shall be concerned with the closed subalgebra

$$
\Lambda_{\gamma}^{+}=\left\{f \in \Lambda_{\gamma}: \widehat{f}(n)=0 \text { for } n<0\right\}
$$

of $\Lambda_{\gamma}$ (where $\widehat{f}(n)$ is the $n$th Fourier coefficient of $f$ ). Since every function in $\Lambda_{\gamma}^{+}$has an extension to a function analytic in the open unit disc $\mathbb{D}$, we

2000 Mathematics Subject Classification: 46J20, 46J15, 30H05.

This work was carried out at Université Bordeaux 1 while the author was holding a TMR Marie Curie postdoctoral grant from the European Commission. 
deduce that

$$
\Lambda_{\gamma}^{+}=\Lambda_{\gamma} \cap \mathcal{A}(\overline{\mathbb{D}}),
$$

where $\mathcal{A}(\overline{\mathbb{D}})$ is the usual disc algebra. Moreover, a function $f$ analytic on $\mathbb{D}$ belongs to $\Lambda_{\gamma}^{+}$if and only if

$$
\left|f^{\prime}(z)\right| \leq C(1-|z|)^{\gamma-1} \quad(z \in \mathbb{D}),
$$

and

$$
\|f\|_{\Lambda_{\gamma}^{+}}=\|f\|_{\infty}+\sup _{z \in \mathbb{D}}\left|f^{\prime}(z)\right|(1-|z|)^{1-\gamma} \quad\left(f \in \Lambda_{\gamma}^{+}\right)
$$

defines an equivalent norm on $\Lambda_{\gamma}^{+}([3$, Theorem 5.1]). In particular, we have $f \in \Lambda_{1}^{+}$if and only if $f^{\prime} \in \mathcal{H}^{\infty}$ (the algebra of bounded analytic functions on $\mathbb{D})$. In passing, we mention that Dyakonov ([4]) has shown that

$$
\|f\|_{\infty}+\sup \left\{\frac{|| f(z)|-| f(w)||}{|z-w|^{\gamma}}: z, w \in \overline{\mathbb{D}}, z \neq w\right\} \quad\left(f \in \Lambda_{\gamma}^{+}\right)
$$

defines an equivalent norm on $\Lambda_{\gamma}^{+}$. This is a remarkable result since this norm only depends on the moduli of the functions. However, for practical purposes the norm $\|\cdot\|_{\Lambda_{\gamma}^{+}}$is easier to estimate.

In this paper, we describe certain closed ideals in $\Lambda_{\gamma}^{+}$by means of zero sets and inner functions. For $f \in \Lambda_{\gamma}^{+}$, let

$$
Z(f)=\{z \in \overline{\mathbb{D}}: f(z)=0\}
$$

be the zero set of $f$ (counting multiplicities on $\mathbb{D}$ ). Also, for a closed ideal $I$ in $\Lambda_{\gamma}^{+}$, let

$$
Z_{I}=\bigcap_{f \in I} Z(f)
$$

be the hull of $I$, let

$$
E_{I}=Z_{I} \cap \mathbb{T}
$$

and let $Q_{I}$ be the greatest common divisor of the inner parts of non-zero functions in $I$ ([6, p. 85]). We shall use the following result of Havin and Shamoyan several times. (See, for instance, [15].)

Theorem 1.1. If $f \in \Lambda_{\gamma}^{+}$and $Q$ is an inner function for which $f / Q$ $\in \mathcal{H}^{\infty}$, then $f / Q \in \Lambda_{\gamma}^{+}$and

$$
\|f / Q\|_{\Lambda_{\gamma}^{+}} \leq C\|f\|_{\Lambda_{\gamma}^{+}} \cdot
$$

In particular, if $f$ belongs to a closed ideal I in $\Lambda_{\gamma}^{+}$, then $f / Q_{I} \in \Lambda_{\gamma}^{+}$.

Recall that a closed set $E \subseteq \mathbb{T}$ is called a Carleson set if

$$
\int_{\mathbb{T}} \log d\left(e^{i \theta}, E\right) d \theta>-\infty
$$


Carleson ([2, Theorem 1]) proved that $E$ is a Carleson set if and only if there exists a function $f \in \Lambda_{\gamma}^{+}$with $E=Z(f)$. In this case

$$
I_{\gamma}(E)=\left\{f \in \Lambda_{\gamma}^{+}: f=0 \text { on } E\right\}
$$

is a closed ideal in $\Lambda_{\gamma}^{+}$with $E_{I_{\gamma}(E)}=E$ and $Q_{I_{\gamma}(E)}=1$. Now, let $Q=B S$ be an inner function, where $B$ is a Blaschke product and $S$ a singular inner function. Let $Z(B)$ be the zeros of $B$ (in $\mathbb{D}$ ) and let $\operatorname{supp}(S)$ be the support of the singular measure on $\mathbb{T}$ that defines $S$. It follows from [9, Theorems 2 and 4] that there exists a function $f \in I_{\gamma}(E)$ with inner factor $Q$ if and only if

$$
\left\{\begin{array}{l}
\int_{\mathbb{T}} \log d\left(e^{i \theta}, E \cup Z(B)\right) d \theta>-\infty, \\
\operatorname{supp}(S) \subseteq E \\
\overline{Z(B)} \backslash Z(B) \subseteq E .
\end{array}\right.
$$

In this case $f / Q \in \Lambda_{\gamma}^{+}$by the previous theorem and

$$
I_{\gamma}(E, Q)=\left\{f \in I_{\gamma}(E): f / Q \in \Lambda_{\gamma}^{+}\right\}
$$

is a closed ideal in $\Lambda_{\gamma}^{+}$with $E_{I_{\gamma}(E, Q)}=E$ and $Q_{I_{\gamma}(E, Q)}=Q$. Clearly, $I_{\gamma}(E, Q)$ is the largest closed ideal $I$ in $\Lambda_{\gamma}^{+}$with $E_{I}=E$ and $Q_{I}=Q$.

For $0<\gamma<1$, our results are motivated by the ideal structure in the little Lipschitz algebra $\lambda_{\gamma}^{+}$, which is the closed subalgebra of $\Lambda_{\gamma}^{+}$of functions $f$ satisfying

$$
|f(z)-f(w)|=o\left(|z-w|^{\gamma}\right)
$$

uniformly as $|z-w| \rightarrow 0$. Matheson ([11]) showed that

$$
I=\left\{f \in \lambda_{\gamma}^{+}: f=0 \text { on } E_{I} \text { and } f / Q_{I} \in \mathcal{H}^{\infty}\right\}=I_{\gamma}\left(E_{I}, Q_{I}\right) \cap \lambda_{\gamma}^{+}
$$

for every closed ideal $I$ in $\lambda_{\gamma}^{+}$. In the non-separable algebra $\Lambda_{\gamma}^{+}$, it is not possible to obtain such a result. This is most easily seen for $\gamma=1$. Let $\chi$ be a character on $\mathcal{H}^{\infty}$ belonging to the fiber at $z=1$, that is, $\chi(\alpha)=1$, where $\alpha$ denotes the function $z \mapsto z$ (see, for example, [6, Chapter 10]). Then

$$
I_{\chi}=\left\{f \in I_{1}(\{1\}): \chi\left(f^{\prime}\right)=0\right\}
$$

is a closed ideal in $\Lambda_{1}^{+}$with $E_{I_{\chi}}=\{1\}$ and $Q_{I_{\chi}}=1$. Moreover, $I_{\chi_{1}} \neq I_{\chi_{2}}$ if $\chi_{1} \neq \chi_{2}$. Similarly, for $0<\gamma<1$, we shall see that there are uncountably many closed ideals $I$ in $\Lambda_{\gamma}^{+}$with $E_{I}=\{1\}$ and $Q_{I}=1$. Nevertheless, we shall obtain certain results about the ideal structure in $\Lambda_{\gamma}^{+}$.

In the algebra $\Lambda_{\gamma}$ on $\mathbb{T}$, Sherbert ([14, Theorem 5.1]) proved that, for a closed set $E \subseteq \mathbb{T}$, the closed ideal

$$
\begin{aligned}
\left\{f \in \Lambda_{\gamma}: f=0 \text { on } E \text { and }|f(z)-f(w)|=o\left(|z-w|^{\gamma}\right)\right. & \\
& \text { as } d(z, E), d(w, E) \rightarrow 0\}
\end{aligned}
$$

is the smallest closed ideal in $\Lambda_{\gamma}$ which has $E$ as hull. We shall prove a 
similar result for $\Lambda_{\gamma}^{+}$. For a Carleson set $E \subseteq \mathbb{T}$, let $J_{\gamma}(E)=\left\{f \in I_{\gamma}(E):|f(z)-f(w)|=o\left(|z-w|^{\gamma}\right)\right.$ as $\left.d(z, E), d(w, E) \rightarrow 0\right\}$.

It is easily seen that $J_{\gamma}(E)$ is a closed ideal in $\Lambda_{\gamma}^{+}$. Also, for a closed set $E \subseteq \mathbb{T}$ and an inner function $Q$ satisfying (2), let

$$
J_{\gamma}(E, Q)=\left\{f \in J_{\gamma}(E): f / Q \in \mathcal{H}^{\infty}\right\} .
$$

It follows from Theorem 1.1 that $J_{\gamma}(E, Q)$ is a closed ideal in $\Lambda_{\gamma}^{+}$, and $E_{J_{\gamma}(E, Q)}=E$ and $Q_{J_{\gamma}(E, Q)}=Q$ by [9, Theorem 4]. The main result in this paper is that the following conjecture holds when $E_{I}$ is countable.

Conjecture. Let $I$ be a closed ideal in $\Lambda_{\gamma}^{+}$. Then $J_{\gamma}\left(E_{I}, Q_{I}\right) \subseteq I$.

The proof of Matheson's result (and of other similar results in separable algebras - see, for instance, [1], [10] and [16]) was to a high extent based on the so-called Carleman transform. (See the next section for the definition.) Apparently, Hedenmalm ([5]) was the first to apply the Carleman transform to a non-separable Banach algebra, when he obtained certain results about the ideal structure in the algebra $\mathcal{H}^{\infty}$.

The proof of our main result uses the Carleman transform and ideas by Bennett and Gilbert ([1]). The Carleman transform of a linear functional $\varphi$ depends only on the restriction of $\varphi$ to the separable subalgebra $\lambda_{\gamma}^{+}$and we therefore find it interesting that it can be used to obtain results about $\Lambda_{\gamma}^{+}$. Moreover, we use a representation of the Carleman transform which is different from the one used in [1], and by following the lines of our proof, one can actually obtain a simpler proof of the main result in [1].

The organization of the paper is as follows. We first obtain some basic facts about the Carleman transform (Section 2) and the ideal $J_{\gamma}(E, Q)$ (Section 3). In Section 4 we prove our main result, and in Section 5 we partially confirm our conjecture for closed ideals $I$ in $\Lambda_{\gamma}^{+}$with $Q_{I}=1$. Finally, in Section 6 we show that the wk$^{*}$ closed ideals in $\Lambda_{\gamma}^{+}$are exactly the ideals $I_{\gamma}(E, Q)$, where the closed set $E \subseteq \mathbb{T}$ and the inner function $Q$ satisfy (2).

2. The Carleman transform. For $\varphi \in\left(\Lambda_{\gamma}^{+}\right)^{*}$, we define the Carleman transform $\Phi$ of $\varphi$ on $\mathbb{C} \backslash \overline{\mathbb{D}}$ by

$$
\Phi(z)=\left\langle(z-\alpha)^{-1}, \varphi\right\rangle \quad(z \in \mathbb{C} \backslash \overline{\mathbb{D}}) .
$$

With $\widehat{\varphi}(n)=\left\langle\alpha^{n}, \varphi\right\rangle$ for $n \in \mathbb{N}_{0}$, we have

$$
\Phi(z)=\sum_{n=0}^{\infty} \widehat{\varphi}(n) z^{-(n+1)} \quad(z \in \mathbb{C} \backslash \overline{\mathbb{D}}) .
$$


For $f \in \Lambda_{\gamma}^{+}$and $0<r<1$, let $f_{r}(z)=f(r z)(z \in \overline{\mathbb{D}})$. For notational convenience, let

$$
\lambda_{1}^{+}=\left\{f \in \Lambda_{1}^{+}: f^{\prime} \in \mathcal{A}(\overline{\mathbb{D}})\right\} .
$$

For $f \in \lambda_{\gamma}^{+}$, it is well known (see, for example, [8, I.2.13]) that $f_{r} \rightarrow f$ in $\lambda_{\gamma}^{+}$as $r \rightarrow 1_{-}$. Hence

$$
\begin{aligned}
\langle f, \varphi\rangle & =\lim _{r \rightarrow 1_{-}}\left\langle f_{r}, \varphi\right\rangle=\lim _{r \rightarrow 1_{-}} \sum_{n=0}^{\infty} \widehat{f}(n) r^{n} \widehat{\varphi}(n) \\
& =\lim _{s \rightarrow 1_{+}} \frac{1}{2 \pi} \int_{\mathbb{T}} f\left(e^{i \theta}\right) e^{i \theta} \Phi\left(s e^{i \theta}\right) d \theta
\end{aligned}
$$

and this was used by Matheson in his proof. However, for $f \in \Lambda_{\gamma}^{+} \backslash \lambda_{\gamma}^{+}$, we do not have $f_{r} \rightarrow f$ in $\Lambda_{\gamma}^{+}$as $r \rightarrow 1_{-}$, so this method does not work in our case.

Let $I$ be a closed ideal in $\Lambda_{\gamma}^{+}$, let

$$
I^{\perp}=\left\{\varphi \in\left(\Lambda_{\gamma}^{+}\right)^{*}:\langle f, \varphi\rangle=0 \text { for every } f \in I\right\}
$$

be the annihilator of $I$ and let $\pi: \Lambda_{\gamma}^{+} \rightarrow \Lambda_{\gamma}^{+} / I$ be the quotient map. Suppose that $\varphi \in I^{\perp}\left(=\left(\Lambda_{\gamma}^{+} / I\right)^{*}\right)$. It is well known that the character space of the algebra $\Lambda_{\gamma}^{+} / I$ equals $Z_{I}$, so the spectrum of $\pi(\alpha)$ equals $Z_{I}$ and the function

$$
\Phi(z)=\left\langle(z-\pi(\alpha))^{-1}, \varphi\right\rangle \quad\left(z \in \mathbb{C} \backslash Z_{I}\right)
$$

thus extends the domain of $\Phi$ to $\mathbb{C} \backslash Z_{I}$.

For $f \in \Lambda_{\gamma}^{+}$and $z \in \mathbb{D}$, define $S_{z} f$ by

$$
\left(S_{z} f\right)(w)= \begin{cases}\frac{f(z)-f(w)}{z-w} & \text { for } w \in \overline{\mathbb{D}} \backslash\{z\}, \\ f^{\prime}(z) & \text { for } w=z .\end{cases}
$$

Then $S_{z} f \in \Lambda_{\gamma} \cap \mathcal{A}(\overline{\mathbb{D}})=\Lambda_{\gamma}^{+}$. It is easily seen that

$$
\left\|(z-\alpha)^{-1}\right\|_{\Lambda_{\gamma}} \leq C|1-| z||^{-(1+\gamma)} \quad(z \in \mathbb{C} \backslash \mathbb{T}),
$$

so we have

$$
\left\|S_{z} f\right\|_{\Lambda_{\gamma}^{+}} \leq C(1-|z|)^{-(1+\gamma)} \quad(z \in \mathbb{D})
$$

We shall often use the following representation of $\Phi$.

Lemma 2.1. Let $I$ be a closed ideal in $\Lambda_{\gamma}^{+}$and let $\varphi \in I^{\perp}$. Then

for $g \in I$.

$$
\Phi(z)=\frac{\left\langle S_{z} g, \varphi\right\rangle}{g(z)} \quad(z \in \mathbb{D} \backslash Z(g))
$$

Proof. For $g \in I$ and $z \in \mathbb{D} \backslash Z(g)$, we have $(z-\alpha) S_{z} g=g(z)-g$ and thus $(z-\pi(\alpha))^{-1}=\pi\left(S_{z} g\right) / g(z)$, so the result follows. 
The normal approach to the Carleman transform (see, for example, [1], [10], [11] and [16]) is to define $\Phi$ on $\mathbb{D} \backslash Z_{I}$ by the expression $\Phi(z)=$ $\left\langle S_{z} g, \varphi\right\rangle / g(z)$ and then show that $\Phi$ extends analytically to $\mathbb{C} \backslash Z_{I}$. With the present definition, we obtained this as an immediate consequence of the general fact from Banach algebra theory that the character space of the algebra $\Lambda_{\gamma}^{+} / I$ equals $Z_{I}$.

The following result is similar to [1, Theorem 2.4].

Lemma 2.2. Let $I$ be a closed ideal in $\Lambda_{\gamma}^{+}$and let $\varphi \in I^{\perp}$. Suppose that $z_{0} \in Z_{I} \cap \mathbb{D}$ is of multiplicity $k$. Then $\Phi$ has a pole of order at most $k$ at $z_{0}$.

Proof. There exist $g \in I$ and $h \in \Lambda_{\gamma}^{+}$with $h\left(z_{0}\right) \neq 0$ such that $g=$ $\left(\alpha-z_{0}\right)^{k} h$. By the previous lemma, we thus have

$$
\left(z-z_{0}\right)^{k} \Phi(z)=\left(z-z_{0}\right)^{k} \frac{\left\langle S_{z} g, \varphi\right\rangle}{g(z)}=\frac{\left\langle S_{z} g, \varphi\right\rangle}{h(z)}
$$

for $z$ in a neighborhood of $z_{0}$, which proves the lemma.

For $\varphi \in\left(\Lambda_{\gamma}^{+}\right)^{*}$ and $f \in \Lambda_{\gamma}^{+}$, we define $\varphi_{f}(=f \varphi) \in\left(\Lambda_{\gamma}^{+}\right)^{*}$ by

$$
\left\langle g, \varphi_{f}\right\rangle=\langle f g, \varphi\rangle \quad\left(g \in \Lambda_{\gamma}^{+}\right) .
$$

If $I$ is a closed ideal in $\Lambda_{\gamma}^{+}$and $\varphi \in I^{\perp}$, then $\varphi_{f} \in I^{\perp}$ for $f \in \Lambda_{\gamma}^{+}$. We denote the Carleman transform of $\varphi_{f}$ by $\Phi_{f}$. Whereas $\Phi$ depends only on the restriction of $\varphi$ to $\lambda_{\gamma}^{+}$, the function $\Phi_{f}$ depends only on the restriction of $\varphi$ to the subalgebra $\lambda_{\gamma}^{+} f$ of $\Lambda_{\gamma}^{+}$. Heuristically, this is the reason why the Carleman transform can be successfully applied to the non-separable algebra $\Lambda_{\gamma}^{+}$.

Lemma 2.3. Let $f \in \Lambda_{\gamma}^{+}$, let $I$ be a closed ideal in $\Lambda_{\gamma}^{+}$and let $\varphi \in I^{\perp}$. Then

$$
\Phi_{f}(z)=f(z) \Phi(z)-\left\langle S_{z} f, \varphi\right\rangle
$$

for $z \in \mathbb{D} \backslash Z_{I}$.

Proof. Let $z \in \mathbb{D} \backslash Z_{I}$ and choose $g \in I$ such that $g(z) \neq 0$. Since $g S_{z} f \in I$, we have

$$
\begin{aligned}
\Phi_{f}(z)-f(z) \Phi(z) & =\frac{\left\langle S_{z} g, \varphi_{f}\right\rangle-f(z)\left\langle S_{z} g, \varphi\right\rangle}{g(z)} \\
& =\frac{\left\langle(f-f(z)) S_{z} g, \varphi\right\rangle}{g(z)}=\frac{\left\langle(g-g(z)) S_{z} f, \varphi\right\rangle}{g(z)}=-\left\langle S_{z} f, \varphi\right\rangle
\end{aligned}
$$

as required.

3. The ideal $J_{\gamma}(E, Q)$. In this section, we prove some basic facts about $J_{\gamma}(E, Q)$. In order to use the characterization (1) of $\Lambda_{\gamma}^{+}$, we need to describe $J_{\gamma}(E)$ in terms of derivatives. 
Proposition 3.1. For a closed set $E \subseteq \mathbb{T}$ and $f \in \Lambda_{\gamma}^{+}$, the following conditions are equivalent:

(a) $f \in J_{\gamma}(E)$.

(b) $f \in I_{\gamma}(E)$ and $\left|f^{\prime}(z)\right|=o\left((1-|z|)^{\gamma-1}\right)$ as $d(z, E) \rightarrow 0$.

Proof. (a) $\Rightarrow($ b). Given $\varepsilon>0$, we choose $\delta>0$ such that $|f(z)-f(w)|<$ $\varepsilon|z-w|^{\gamma}$ for $z, w \in \overline{\mathbb{D}}$ with $d(z, E), d(w, E)<\delta$. Let $z \in \mathbb{D}$ with $d(z, E)<\delta / 2$ and let $r=1-|z|<\delta / 2$. Then $d(w, E)<\delta$ for $|w-z|=r$, so Cauchy's formula

$$
f^{\prime}(z)=\frac{1}{2 \pi i} \oint_{|w-z|=r} \frac{f(w)-f(z)}{(w-z)^{2}} d w
$$

shows that $\left|f^{\prime}(z)\right|<\varepsilon r^{\gamma-1}$ as required.

(b) $\Rightarrow\left(\right.$ a). Let $\varepsilon>0$ and choose $\delta_{1}>0$ such that $\left|f^{\prime}(z)\right|<\varepsilon(1-|z|)^{\gamma-1}$ for $z \in \mathbb{D}$ with $d(z, E)<\delta_{1}$. Choose $\delta_{2}>0$ such that $|f(z)|<\varepsilon \delta_{1}^{\gamma}$ for $z \in \overline{\mathbb{D}}$ with $d(z, E)<\delta_{2}$ and let $\delta=\min \left\{\delta_{1}, \delta_{2}\right\}$. Let $z_{1}, z_{2} \in \overline{\mathbb{D}}$ with $d\left(z_{k}, E\right)<\delta / 3(k=1,2)$. If $\left|z_{2}-z_{1}\right| \geq \delta_{1} / 3$, then

$$
\left|f\left(z_{2}\right)-f\left(z_{1}\right)\right|<2 \varepsilon \delta_{1}^{\gamma} \leq 2 \cdot 3^{\gamma} \varepsilon\left|z_{2}-z_{1}\right|^{\gamma},
$$

so we may assume that $\left|z_{2}-z_{1}\right|<\delta_{1} / 3$. With $z_{k}=r_{k} e^{i \theta_{k}}(k=1,2)$, we may also assume that $r_{1} \geq r_{2}$ and that $0 \leq \theta_{2}-\theta_{1} \leq \pi$. First, suppose that $\left|z_{2}-z_{1}\right| \leq 1-r_{1}$. Since $d(w, E)<\delta$ and $|w| \leq r_{1}$ for every point $w$ on the line segment from $z_{1}$ to $z_{2}$, we deduce that

$$
\left|f\left(z_{2}\right)-f\left(z_{1}\right)\right|<\left|z_{2}-z_{1}\right| \varepsilon\left(1-r_{1}\right)^{\gamma-1} \leq \varepsilon\left|z_{2}-z_{1}\right|^{\gamma} .
$$

Now, suppose that $\left|z_{2}-z_{1}\right| \geq 1-r_{1}$. Let $\varrho=1-\left|z_{2}-z_{1}\right|$ and let $\Gamma$ be the curve

$$
\begin{aligned}
\Gamma= & \left\{r e^{i \theta_{1}}: \varrho \leq r \leq r_{1}\right\} \cup\left\{\varrho e^{i \theta}: \theta_{1} \leq \theta \leq \theta_{2}\right\} \\
& \cup\left\{r e^{i \theta_{2}}: r \text { is between } \varrho \text { and } r_{2}\right\} .
\end{aligned}
$$

Then $d(w, E)<\delta$ for $w \in \Gamma$, so

$$
\left|f\left(\varrho e^{i \theta_{1}}\right)-f\left(z_{1}\right)\right|<\varepsilon \int_{\varrho}^{1}(1-r)^{\gamma-1} d r=(\varepsilon / \gamma)\left|z_{2}-z_{1}\right|^{\gamma} .
$$

Similarly, if $\varrho \leq r_{2}$, then

$$
\left|f\left(z_{2}\right)-f\left(\varrho e^{i \theta_{2}}\right)\right|<\varepsilon\left|z_{2}-z_{1}\right|^{\gamma} .
$$

If $\varrho \geq r_{2}$, then

$$
\begin{aligned}
\left|f\left(z_{2}\right)-f\left(\varrho e^{i \theta_{2}}\right)\right| & <\varepsilon \int_{r_{2}}^{\varrho}(1-r)^{\gamma-1} d r \leq(\varepsilon / \gamma)\left(\left(1-r_{2}\right)^{\gamma}-\left(1-r_{1}\right)^{\gamma}\right) \\
& \leq \varepsilon\left(r_{1}-r_{2}\right)^{\gamma} \leq \varepsilon\left|z_{2}-z_{1}\right|^{\gamma} .
\end{aligned}
$$


Moreover,

$$
\left|f\left(\varrho e^{i \theta_{2}}\right)-f\left(\varrho e^{i \theta_{1}}\right)\right|<\varepsilon\left(\theta_{2}-\theta_{1}\right)(1-\varrho)^{\gamma-1} \leq C \varepsilon\left|z_{2}-z_{1}\right|^{\gamma},
$$

so we obtain

$$
\left|f\left(z_{2}\right)-f\left(z_{1}\right)\right|<(C+2) \varepsilon\left|z_{2}-z_{1}\right|^{\gamma}
$$

as required.

For $\gamma=1$, the previous proposition takes the following form. Let

$$
\mathcal{H}_{E}^{\infty}=\left\{f \in \mathcal{H}^{\infty}: f^{\prime}(z) \rightarrow 0 \text { as } d(z, E) \rightarrow 0\right\}
$$

for a closed set $E \subseteq \mathbb{T}$.

Corollary 3.2. For a closed set $E \subseteq \mathbb{T}$ and $f \in \Lambda_{1}^{+}$, we have $f \in J_{1}(E)$ if and only if $f \in I_{1}(E)$ and $f^{\prime} \in \mathcal{H}_{E}^{\infty}$.

We shall use the notation

$$
J_{\gamma, 0}=J_{\gamma}(\{1\}), \quad I_{\gamma, 0}=I_{\gamma}(\{1\}) .
$$

Also, for $s>0$, let $\psi_{-s}$ be the singular inner function defined by

$$
\psi_{-s}(z)=\exp \left(-s \frac{1+z}{1-z}\right) \quad(z \in \overline{\mathbb{D}} \backslash\{1\})
$$

and write

$$
J_{\gamma, s}=J_{\gamma}\left(\{1\}, \psi_{-s}\right), \quad I_{\gamma, s}=I_{\gamma}\left(\{1\}, \psi_{-s}\right) .
$$

For $n \in \mathbb{N}$, let

$$
K_{n}=\frac{1-\alpha}{1+1 / n-\alpha} .
$$

For many separable Banach algebras of analytic functions on $\mathbb{D}$, it is well known that the sequence $\left(K_{n}\right)$ is an approximate identity for the maximal ideal of functions vanishing at $z=1$. In our case, the local condition at $z=1$ imposed on functions in $J_{\gamma, 0}$ enables us to prove the following result.

Lemma 3.3. For $f \in J_{\gamma, 0}$, we have $K_{n} f \rightarrow f$ in $\Lambda_{\gamma}^{+}$as $n \rightarrow \infty$. In particular, for $\gamma<1$, the sequence $\left(K_{n}\right)$ is an approximate identity for the ideal $J_{\gamma, 0}$.

Proof. Let $f \in J_{\gamma, 0}$ and let $p_{n}=1-K_{n}=n^{-1}(1+1 / n-\alpha)^{-1}(n \in \mathbb{N})$. Since $p_{n} \rightarrow 0$ uniformly on compact subsets of $\overline{\mathbb{D}} \backslash\{1\}$ as $n \rightarrow \infty$, it follows that

$$
\sup _{z \in \mathbb{D}}\left|p_{n}(z) f^{\prime}(z)\right|(1-|z|)^{1-\gamma} \rightarrow 0
$$


as $n \rightarrow \infty$. Also,

$$
\begin{aligned}
\left|p_{n}^{\prime}(z) f(z)\right|(1-|z|)^{1-\gamma} & \leq \varepsilon(|1-z|)\left|\frac{1-z}{n(1+1 / n-z)^{2}}\right| \\
& =\varepsilon(|1-z|)\left|\frac{1-z}{1+1 / n-z}\right|\left|\frac{1}{n(1+1 / n-z)}\right| \\
& \leq \varepsilon(|1-z|) \quad(z \in \overline{\mathbb{D}}),
\end{aligned}
$$

where $\varepsilon(t) \rightarrow 0$ as $t \rightarrow 0$. Since

$$
\frac{1-z}{n(1+1 / n-z)^{2}} \rightarrow 0
$$

uniformly on compact subsets of $\overline{\mathbb{D}} \backslash\{1\}$ as $n \rightarrow \infty$, it thus follows that

$$
\sup _{z \in \mathbb{D}}\left|p_{n}^{\prime}(z) f(z)\right|(1-|z|)^{1-\gamma} \rightarrow 0
$$

as $n \rightarrow \infty$. Hence $p_{n} f \rightarrow 0$ in $\Lambda_{\gamma}^{+}$as $n \rightarrow \infty$.

We finish this section with a description of the ideals $J_{\gamma, s}$ in terms of generators.

LEMMA 3.4. Let $\beta, s>0$ and let $f=(1-\alpha)^{\beta} \psi_{-s}$. Then $f \in \Lambda_{\gamma}^{+}$if and only if $\beta \geq 2 \gamma$ and $f \in J_{\gamma, s}$ if and only if $\beta>2 \gamma$.

Proof. We have

$$
f^{\prime}=-\beta(1-\alpha)^{\beta-1} \psi_{-s}-2 s(1-\alpha)^{\beta-2} \psi_{-s} .
$$

For $z \in \mathbb{D}$, we write $1-z=r e^{i \theta}$. Then $1-|z|^{2}=r(2 \cos \theta-r)$, so $2 \cos \theta>$ $r>0$. Also,

$$
\operatorname{Re}\left(\frac{1+z}{1-z}\right)=\frac{2 \cos \theta}{r}-1
$$

SO

$$
\begin{aligned}
|1-z|^{\beta-2}\left|\psi_{-s}(z)\right| & \left(1-|z|^{2}\right)^{1-\gamma} \\
& =r^{\beta-2} \exp \left(-s\left(\frac{2 \cos \theta}{r}-1\right)\right)(r(2 \cos \theta-r))^{1-\gamma} \\
& =r^{\beta-2 \gamma} \exp \left(-s\left(\frac{2 \cos \theta}{r}-1\right)\right)\left(\frac{2 \cos \theta}{r}-1\right)^{1-\gamma}
\end{aligned}
$$

and the result follows.

Proposition 3.5. (a) For $\beta>\gamma$, we have

$$
J_{\gamma, 0}=\overline{\Lambda_{\gamma}^{+}(1-\alpha)^{\beta}} \text {. }
$$

(b) For $s>0$ and $\beta>2 \gamma$, we have

$$
J_{\gamma, s}=\overline{\Lambda_{\gamma}^{+}(1-\alpha)^{\beta} \psi_{-s}} .
$$


Proof. (a) Since $(1-\alpha)^{\beta} \in J_{\gamma, 0}$, we have the inclusion $\overline{\Lambda_{\gamma}^{+}(1-\alpha)^{\beta}}$ $\subseteq J_{\gamma, 0}$. Moreover, it follows from Lemma 3.3 that $J_{\gamma, 0}=\overline{J_{\gamma, 0}(1-\alpha)}$ and thus $J_{\gamma, 0} \subseteq \overline{\Lambda_{\gamma}^{+}(1-\alpha)^{m}}$ for $m \in \mathbb{N}$, which proves the reverse inclusion.

(b) It follows from the previous lemma that

$$
\overline{\Lambda_{\gamma}^{+}(1-\alpha)^{\beta} \psi_{-s}} \subseteq J_{\gamma, s} .
$$

Conversely, let $f \in J_{\gamma, s}$. By Lemma 3.3 , we have $K_{n} f \rightarrow f$ in $\Lambda_{\gamma}^{+}$as $n \rightarrow \infty$. Fix $n \in \mathbb{N}$ and let $g=K_{n} f$. Let $0<a<1$ and let

$$
T^{\varepsilon}(z)=\exp \left(-\varepsilon(1-z)^{-a}\right) \quad(z \in \overline{\mathbb{D}}) .
$$

Then $|T| \leq 1$ on $\overline{\mathbb{D}}$ and

$$
T^{\prime}(z)=-a(1-z)^{-(a+1)} T(z) \quad(z \in \overline{\mathbb{D}}),
$$

so $\left(T^{\varepsilon}\right)$ is a semigroup of outer functions in $\Lambda_{\gamma}^{+}$. (In Section 6 , we shall make use of a more general version of this semigroup.) Moreover,

$$
\left(T^{\varepsilon} g\right)^{\prime}=T^{\varepsilon} g^{\prime}+\varepsilon\left(T^{\prime} / T\right) T^{\varepsilon} g \quad(\varepsilon>0) .
$$

Since $T^{\varepsilon} \rightarrow 1$ uniformly on compact subsets of $\overline{\mathbb{D}} \backslash\{1\}$ and since $|g(z)| \leq$ $C|1-z|^{\gamma+1}$ for $z \in \overline{\mathbb{D}}$, we deduce that $T^{\varepsilon} g \rightarrow g$ in $\Lambda_{\gamma}^{+}$as $\varepsilon \rightarrow 0$. Finally, using (a), we choose a sequence $\left(g_{m}\right)$ in $\Lambda_{\gamma}^{+}$such that

$$
g_{m}(1-\alpha)^{\beta} \rightarrow g / \psi_{-s}
$$

in $\Lambda_{\gamma}^{+}$as $m \rightarrow \infty$. It is easily seen that $T^{\varepsilon} \psi_{-s} \in \Lambda_{\gamma}^{+}$, so

$$
T^{\varepsilon} g_{m}(1-\alpha)^{\beta} \psi_{-s} \rightarrow T^{\varepsilon} g
$$

in $\Lambda_{\gamma}^{+}$as $m \rightarrow \infty$ for $\varepsilon>0$, and it follows that $f \in \overline{\Lambda_{\gamma}^{+}(1-\alpha)^{\beta} \psi_{-s}}$.

4. Ideals with countable hull. Our main aim in this paper is to prove the following result.

THEOREM 4.1. We have

$$
J_{\gamma}\left(E_{I}, Q_{I}\right) \subseteq I
$$

for every closed ideal $I$ in $\Lambda_{\gamma}^{+}$for which $E_{I}$ is countable.

Before proceeding to the proof of the theorem, we present a few consequences. It follows from Theorem 1.1 that if $f \in I_{\gamma}(E, Q)$, then $f / Q \in$ $I_{\gamma}(E)$. We do not know whether the corresponding result for $J_{\gamma}(E, Q)$ holds in general, but for $E$ countable it follows easily from the theorem.

Corollary 4.2. Suppose that a closed set $E \subseteq \mathbb{T}$ and an inner function $Q$ satisfy (2) and that $E$ is countable. If $f \in J_{\gamma}(E, Q)$, then $f / Q \in$ $J_{\gamma}(E)$. 
Proof. Consider the closed ideal

$$
I=\left\{f \in J_{\gamma}(E, Q): f / Q \in J_{\gamma}(E)\right\}
$$

in $\Lambda_{\gamma}^{+}$. We have $E_{I}=E$ and $Q_{I}=Q$, so the previous theorem entails that $J_{\gamma}(E, Q) \subseteq I$ and the conclusion follows.

For $\gamma=1$, Theorem 4.1 can be restated as follows with the use of Corollary 3.2 .

Corollary 4.3. Let I be a closed ideal $I$ in $\Lambda_{1}^{+}$and suppose that $E_{I}$ is countable. Then

$$
\left\{f \in I_{1}\left(E_{I}, Q_{I}\right): f^{\prime} \in \mathcal{H}_{E_{I}}^{\infty}\right\} \subseteq I .
$$

For the primary ideals, more can be said.

Corollary 4.4. Let $s \geq 0$. The closed ideals $I$ in $\Lambda_{\gamma}^{+}$with $E_{I}=\{1\}$ and $Q_{I}=\psi_{-s}$ are exactly the closed subspaces $I$ of $\Lambda_{\gamma}^{+}$with

$$
J_{\gamma, s} \subseteq I \subseteq I_{\gamma, s} .
$$

Proof. Let $I$ be a closed subspace of $\Lambda_{\gamma}^{+}$with $J_{\gamma, s} \subseteq I \subseteq I_{\gamma, s}$. For $f \in \Lambda_{\gamma}^{+}$ and $g \in I$, we have

$$
(f-f(1)) g \in I_{\gamma, 0} \cdot I_{\gamma, s} \subseteq J_{\gamma, s} \subseteq I,
$$

so $f g \in I$ and the result follows.

In his paper [5] on the ideal structure in $\mathcal{H}^{\infty}$, Hedenmalm stated the following result, which is now easily deduced from our results.

Corollary 4.5. Let $I$ be a closed ideal in $\Lambda_{1}^{+}$with $E_{I}=\{1\}$ and $Q_{I}=1$. Then there is a closed subspace $\mathcal{Z}$ in $\mathcal{H}^{\infty}$ containing $\mathcal{H}_{\{1\}}^{\infty}$ such that

$$
I=\left\{f \in I_{1}(\{1\}): f^{\prime} \in \mathcal{Z}\right\} .
$$

Conversely, every such set $I$ is a closed ideal in $\Lambda_{1}^{+}$with $E_{I}=\{1\}$ and $Q_{I}=1$.

Proof. For $f \in I_{1}(\{1\})$, we have $\|f\|_{\infty} \leq 2\left\|f^{\prime}\right\|_{\infty}$, so $f \mapsto\left\|f^{\prime}\right\|_{\infty}$ defines a norm on $I_{1}(\{1\})$ which is equivalent to the $\Lambda_{1}^{+}$norm. Hence $I \mapsto I^{\prime}=$ $\left\{f^{\prime}: f \in I\right\}$ defines a bijective correspondence between the closed subspaces $I$ in $\Lambda_{1}^{+}$with $J_{1}(\{1\}) \subseteq I \subseteq I_{1}(\{1\})$ and the closed subspaces $\mathcal{Z}$ in $\mathcal{H}^{\infty}$ with $\mathcal{H}_{\{1\}}^{\infty} \subseteq \mathcal{Z}$, so the result follows from the previous corollary.

Finally, we shall show that there are uncountably many closed ideals between $J_{\gamma, s}$ and $I_{\gamma, s}$.

LEMMA 4.6. Let $f_{s}=(1-\alpha)^{2 \gamma} \psi_{-s}(s>0)$. For $0<t_{0}<s_{0}$, we have

$$
\left\|f_{s}-f_{t}\right\|_{\Lambda_{\gamma}^{+} / J_{\gamma, 0}} \geq C
$$

for $t_{0} \leq t<s \leq s_{0}$. 
Proof. We have

$$
\|f\|_{\Lambda_{\gamma}^{+} / J_{\gamma, 0}} \geq \limsup _{z \rightarrow 1}\left|f^{\prime}(z)\right|(1-|z|)^{1-\gamma}
$$

for $f \in \Lambda_{\gamma}^{+}$. Also,

$$
f_{s}^{\prime}=-2 \gamma(1-\alpha)^{2 \gamma-1} \psi_{-s}-2 s(1-\alpha)^{2 \gamma-2} \psi_{-s},
$$

so

$$
\begin{aligned}
\left\|f_{s}-f_{t}\right\|_{\Lambda_{\gamma}^{+} / J_{\gamma, 0}} \geq & \limsup _{z \rightarrow 1} \mid 2(s-t)(1-z)^{2 \gamma-2} \psi_{-s}(z) \\
& +2 t(1-z)^{2 \gamma-2}\left(\psi_{-s}(z)-\psi_{-t}(z)\right) \mid(1-|z|)^{1-\gamma} .
\end{aligned}
$$

As in the proof of Lemma 3.4, we write $1-z=r e^{i \theta}$ for $z \in \mathbb{D}$. Then

$$
\operatorname{Im}\left(\frac{1+z}{1-z}\right)=\frac{2 \sin \theta}{r},
$$

so there exists a sequence $\left(z_{n}\right)$ tending to 1 such that

$$
\operatorname{Im}\left(\frac{1+z_{n}}{1-z_{n}}\right)=\frac{(2 n+1) \pi}{s-t}
$$

and thus

$$
\left|\psi_{-(s-t)}\left(z_{n}\right)-1\right| \geq 1-\operatorname{Re} \psi_{-(s-t)}\left(z_{n}\right) \geq 1 .
$$

It thus follows from the proof of Lemma 3.4 that

$$
\limsup _{n \rightarrow \infty}\left|\left(1-z_{n}\right)^{2 \gamma-2}\left(\psi_{-s}\left(z_{n}\right)-\psi_{-t}\left(z_{n}\right)\right)\right|\left(1-\left|z_{n}\right|\right)^{1-\gamma} \geq C .
$$

Hence there exists $\delta>0$ such that

$$
\left\|f_{s}-f_{t}\right\|_{\Lambda_{\gamma}^{+} / J_{\gamma, 0}} \geq t_{0} C
$$

for $0<s-t<\delta$ and the result follows.

Corollary 4.7. For $s \geq 0$, there are uncountably many closed ideals $I$ in $\Lambda_{\gamma}^{+}$with $J_{\gamma, s} \subseteq I \subseteq I_{\gamma, s}$.

Proof. The inclusion map $\iota: I_{\gamma, s} \rightarrow I_{\gamma, 0}$ induces a bounded linear map $\tau: I_{\gamma, s} / J_{\gamma, s} \rightarrow I_{\gamma, 0} / J_{\gamma, 0}$. Since $f_{t} \in I_{\gamma, s}$ for $t \geq s$, we deduce from the previous lemma that $I_{\gamma, s} / J_{\gamma, s}$ is non-separable, so the result follows from Corollary 4.4.

We now turn to the proof of Theorem 4.1. Recall the following definitions (with a few modifications) from [1]:

$H_{+}$: consists of the analytic functions $f$ on $\mathbb{D}$ for which $|f(z)| \leq C(1-|z|)^{-N}$ for $z \in \mathbb{D}$ for some $N \in \mathbb{N}$,

$H_{-}$: consists of the analytic functions $f$ on $\mathbb{C} \backslash \overline{\mathbb{D}}$ with $|f(z)| \leq C(|z|-1)^{-N}$ for $z \in \mathbb{C} \backslash \overline{\mathbb{D}}$ for some $N \in \mathbb{N}$ and $f(z) \rightarrow 0$ as $|z| \rightarrow \infty$, 
$\mathcal{G}$ : consists of the analytic functions $f$ on $\mathbb{C} \backslash \mathbb{T}$ for which $f \in H_{-}$on $\mathbb{C} \backslash \overline{\mathbb{D}}$ and $f=g / h$ with $g \in H_{+}$and $h \in \mathcal{H}^{\infty}$ on $\mathbb{D}$.

The following result as well as its proof are similar to [1, Theorem 4.3].

Proposition 4.8. Let $I$ be a closed ideal in $\Lambda_{\gamma}^{+}$and let $\varphi \in I^{\perp}$. If $f \in J_{\gamma}\left(E_{I}, Q_{I}\right)$, then $\Phi_{f}$ does not have any isolated singularities.

Proof. It follows from Lemma 2.2 that $Q_{I} \Phi$ and thus $f \Phi$ is analytic on $\mathbb{D}$. Hence $\Phi_{f}$ is analytic on $\mathbb{D}$ by Lemma 2.3 , so the singularities of $\Phi_{f}$ belong to $Z_{I} \cap \mathbb{T}=E_{I}$. Moreover, by Lemmas 2.1 and 2.3 , we have

$$
\Phi_{f}(z)=\frac{(f(z) / Q(z))\left\langle S_{z} g, \varphi\right\rangle-(g(z) / Q(z))\left\langle S_{z} f, \varphi\right\rangle}{g(z) / Q(z)} \quad(z \in \mathbb{D} \backslash Z(g))
$$

for $g \in I$. From (3) and (4), we thus deduce that $\Phi_{f} \in \mathcal{G}$, so it follows from $\left[1\right.$, Theorem 3.2(ii)] that any isolated singularity of $\Phi_{f}$ is a pole.

Suppose that $\Phi_{f}$ has a pole of order $p$ at (say) $z=1$, so that the function $\Psi$ defined by

$$
\Psi=(1-\alpha)^{p} \Phi_{f}
$$

is analytic in a neighborhood $U$ of 1 and $a=\Psi(1) \neq 0$. Since $f \in J_{\gamma, 0}$, we have $K_{n} f \rightarrow f$ in $\Lambda_{\gamma}^{+}$as $n \rightarrow \infty$ by Lemma 3.3. Moreover, $K_{n} \in \lambda_{\gamma}^{+}$and the polynomials are dense in $\lambda_{\gamma}^{+}$, so there exists a sequence $\left(p_{n}\right)$ of polynomials with $p_{n}(1)=1$ and $p_{n} f \rightarrow 0$ in $\Lambda_{\gamma}^{+}$as $n \rightarrow \infty$. Let $\varphi_{n}=\varphi_{p_{n} f}$ and let $\Phi_{n}$ be the Carleman transform of $\varphi_{n}$. Since $\varphi_{n}=\left(\varphi_{f}\right)_{p_{n}}$, it follows from Lemma 2.3 that

$$
\Phi_{n}(z)=p_{n}(z) \Phi_{f}(z)-\left\langle S_{z} p_{n}, \varphi_{f}\right\rangle \quad\left(z \in \mathbb{D} \backslash Z_{I}\right)
$$

and $q_{n}(z)=\left\langle S_{z} p_{n}, \varphi_{f}\right\rangle$ is a polynomial in $z$. Combining (5) and (6), we obtain

$$
(1-\alpha)^{p} \Phi_{n}=p_{n} \Psi-(1-\alpha)^{p} q_{n}
$$

on $U$, so the function $\Psi_{n}$ defined by $\Psi_{n}=(1-\alpha)^{p} \Phi_{n}$ is analytic in $U$ and $\Psi_{n}(1)=a$.

Choose a circle $\Gamma$ centered at 1 and contained in $U$ and a function $g \in I$ such that $g(z) \neq 0$ for $z \in \Gamma \cap \overline{\mathbb{D}}$. We have

$$
\left\|\varphi_{n}\right\| \leq\left\|p_{n} f\right\|_{\Lambda_{\gamma}^{+}} \cdot\|\varphi\| \rightarrow 0
$$

as $n \rightarrow \infty$, so

$$
\left|\Phi_{n}(z)\right| \leq C(1-|z|)^{-(\gamma+1)} \quad(z \in \Gamma \cap \mathbb{D})
$$

by (4) and

$$
\left|\Phi_{n}(z)\right| \leq C(|z|-1)^{-(\gamma+1)} \quad(z \in \Gamma \backslash \overline{\mathbb{D}})
$$

by (3). It thus follows from the proof of [8, Lemma VI.8.3] that the sequence $\left(\Psi_{n}\right)$ is uniformly bounded on some disc centered at 1 . By $(7)$, we have $\Phi_{n} \rightarrow 0$ pointwise on $\mathbb{C} \backslash Z_{I}$ as $n \rightarrow \infty$ and thus $\Psi_{n} \rightarrow 0$ pointwise on $\Gamma$ 
as $n \rightarrow \infty$. Hence $\Psi_{n}(1) \rightarrow 0$ as $n \rightarrow \infty$ by Cauchy's integral formula and Lebesgue's dominated convergence theorem, contradicting $\Psi_{n}(1)=a \neq 0$.

Proof of Theorem 4.1. Let $I$ be a closed ideal in $\Lambda_{\gamma}^{+}$, let $\varphi \in I^{\perp}$ and let $f \in J_{\gamma}\left(E_{I}, Q_{I}\right)$. We will use the same transfinite induction as in $[1$, p. 17] to prove that $\Phi_{f}$ is entire. Let $L_{0}=E_{I}$ and inductively define $L_{\sigma}$ for any ordinal $\sigma$ in the following way: If $\sigma=\tau+1$ is not a limit ordinal, we define $L_{\sigma}$ to be the set of limit points of $L_{\tau}$, and if $\sigma$ is a limit ordinal, we let $L_{\sigma}=\bigcap_{\tau<\sigma} L_{\tau}$. If $z_{0}$ is a singularity of $\Phi_{f}$, then $z_{0} \in E_{I}=L_{0}$. Suppose that we have shown that $z_{0} \in L_{\tau}$ for every ordinal $\tau<\sigma$. If $\sigma=\tau+1$ is not a limit ordinal, then $L_{\sigma} \backslash L_{\tau}$ consists of isolated points, so it follows from the previous proposition that $z_{0} \in L_{\sigma}$. The same conclusion clearly holds if $\sigma$ is a limit ordinal, so we conclude that $z_{0} \in L_{\sigma}$ for every ordinal $\sigma$. However, $L_{0}$ contains no perfect subsets, so $L_{\sigma} \subset L_{\tau}$ for every non-limit ordinal $\sigma=\tau+1$, and it follows that there exists a first ordinal $\sigma_{0}$ such that $L_{\sigma_{0}}$ is empty. This contradicts our earlier conclusion $z_{0} \in L_{\sigma_{0}}$. Consequently, $\Phi_{f}$ does not have any singularities, so $\Phi_{f}$ is entire. Hence $\Phi_{f}=0$ and since $\operatorname{span}\left\{(z-\alpha)^{-1}: z \in \mathbb{C} \backslash \overline{\mathbb{D}}\right\}$ is dense in $\lambda_{\gamma}^{+}$, this is equivalent to $\varphi_{f}=0$ on $\lambda_{\gamma}^{+}$. Consequently,

$$
\langle f, \varphi\rangle=\left\langle 1, \varphi_{f}\right\rangle=0
$$

and since $\varphi \in I^{\perp}$ was arbitary, we conclude that $f \in I$.

For closed ideals with finite hull, we shall now give a proof of Theorem 4.1 which is more constructive and does not depend on Proposition 4.8. For simplicity, we consider only closed ideals $I$ in $\Lambda_{\gamma}^{+}$with $Z_{I}=\{1\}$. For $\gamma<1$ and $Q_{I}=1$, the main idea in the proof is to show that if $\varphi \in I^{\perp}$, then $\langle f, \varphi\rangle=a f(1)$ for $f \in \lambda_{\gamma}^{+}$for some $a \in \mathbb{C}$ (and similarly for $\gamma=1$ ).

Proof of Theorem 4.1 when $Z_{I}=\{1\}$. First, suppose that $Q_{I}=1$. For $\varphi \in I^{\perp}$, we have

$$
|\Phi(z)| \leq C(|z|-1)^{-(1+\gamma)} \quad(z \in \mathbb{C} \backslash \overline{\mathbb{D}})
$$

by (3). Moreover, for $g \in I$ and $z \in \mathbb{D}$, it follows from (4) and Lemma 2.1 that

$$
|g(z) \Phi(z)| \leq C(1-|z|)^{-(1+\gamma)} \quad(z \in \mathbb{D}) .
$$

Hence $\Phi$ has a pole at $z=1$ by [1, Theorem 3.2(ii)]. We first consider the case where $0<\gamma<1$. Then $z=1$ is a simple pole of $\Phi$ by (8) and since $\Phi(z) \rightarrow 0$ as $|z| \rightarrow \infty$, we deduce that

$$
\Phi(z)=a(z-1)^{-1} \quad(z \in \mathbb{C} \backslash\{1\})
$$

for some $a \in \mathbb{C}$. Let $\delta_{1} \in\left(\Lambda_{\gamma}^{+}\right)^{*}$ denote the point evaluation at $z=1$. Then

$$
\left\langle(z-\alpha)^{-1}, \delta_{1}\right\rangle=(z-1)^{-1} \quad(z \in \mathbb{C} \backslash \overline{\mathbb{D}}),
$$


so $\varphi=a \delta_{1}$ on the closed span of $\left\{(z-\alpha)^{-1}:|z|>1\right\}$, that is, on $\lambda_{\gamma}^{+}$. In particular, $\langle 1-\alpha, \varphi\rangle=0$. The Hahn-Banach theorem thus implies that $1-\alpha \in I$, so $J_{\gamma, 0} \subseteq I$ by Proposition 3.5. For $\gamma=1$, the same method works with the following changes. From (8), we deduce that $\Phi$ has a pole of order 2 at $z=1$, so

$$
\Phi(z)=a(z-1)^{-1}+b(z-1)^{-2} \quad(z \in \mathbb{C} \backslash\{1\})
$$

for some $a, b \in \mathbb{C}$. On $\lambda_{1}^{+}$, we define $\delta_{1}^{\prime}$ by $\left\langle g, \delta_{1}^{\prime}\right\rangle=g^{\prime}(1)\left(g \in \lambda_{1}^{+}\right)$. Then

$$
\left\langle(z-\alpha)^{-1}, \delta_{1}^{\prime}\right\rangle=(z-1)^{-2} \quad(z \in \mathbb{C} \backslash \overline{\mathbb{D}}),
$$

so $\varphi=a \delta_{1}+b \delta_{1}^{\prime}$ on $\lambda_{1}^{+}$. In particular, $\left\langle(1-\alpha)^{2}, \varphi\right\rangle=0$, so $J_{1,0} \subseteq I$ by Proposition 3.5.

Now, suppose that $Q_{I}=\psi_{-s}$ for some $s>0$. We have $(1-\alpha)^{2} \psi_{-s} \in \Lambda_{\gamma}^{+}$ and the division ideal

$$
\widetilde{I}=\left\{f \in I_{\gamma}(\{1\}):(1-\alpha)^{2} \psi_{-s} f \in I\right\}
$$

satisfies $E_{\widetilde{I}}=\{1\}$ and $Q_{\widetilde{I}}=1$, so $J_{\gamma, 0} \subseteq \widetilde{I}$ by the first part of the proof. Since $(1-\alpha)^{2} \in J_{\gamma, 0}$, we thus have $(1-\alpha)^{4} \psi_{-s} \in I$, so the conclusion follows from Proposition 3.5.

5. Ideals with $Q_{I}=1$. Our aim in this section is to prove the following result.

Theorem 5.1. Let $E \subseteq \mathbb{T}$ be a Carleson set and let $F \in J_{\gamma}(E)$ be an outer function with $Z(F)=E$. Then

$$
\overline{\Lambda_{\gamma}^{+} F}=J_{\gamma}(E) \text {. }
$$

Remarks. (1) We do not know whether a closed ideal $I$ in $\Lambda_{\gamma}^{+}$with $Q_{I}=1$ necessarily contains an outer function $F$ with $Z(F)=E_{I}$. However, if this is the case, then the theorem verifies our conjecture for this class of closed ideals. This is seen as follows: Let $H \in J_{\gamma}\left(E_{I}\right)$ be an outer function with $Z(H)=E_{I}$. Then $F H \in I \cap J_{\gamma}\left(E_{I}\right)$ and $Z(F H)=E_{I}$, so it follows from the theorem that

$$
J_{\gamma}\left(E_{I}\right)=\overline{\Lambda_{\gamma}^{+} F H} \subseteq \overline{\Lambda_{\gamma}^{+} F} \subseteq I
$$

as required.

(2) We do not know how to prove a version of the theorem for the ideals $J_{\gamma}(E, Q)$ with $Q \neq 1$.

For a closed set $E \subseteq \mathbb{T}$ and $p \in \mathbb{N}$, let

$$
I_{\gamma}^{p}(E)=\left\{f \in \Lambda_{\gamma}^{+}:|f(z)| \leq C d(z, E)^{p}(z \in \mathbb{T})\right\} .
$$

For $f \in I_{\gamma}^{p}(E)$, we have $|f(z)| \leq C|z-w|^{p}(z \in \mathbb{T}, w \in E)$ and since $(\alpha-w)^{p}$ is outer, this holds for $z \in \overline{\mathbb{D}}$, so it follows that $|f(z)| \leq C d(z, E)^{p}(z \in \overline{\mathbb{D}})$.

Theorem 5.1 is an immediate consequence of the following two results. 
Proposition 5.2. Let $E \subseteq \mathbb{T}$ be a Carleson set, let $F \in J_{\gamma}(E)$ be an outer function with $Z(F)=E$ and let $p \in \mathbb{N}$ with $p>2 \gamma$. Then

$$
J_{\gamma}(E) \cap I_{\gamma}^{p}(E) \subseteq \overline{\Lambda_{\gamma}^{+} F} .
$$

Proposition 5.3. Let $E \subseteq \mathbb{T}$ be a Carleson set and let $p \in \mathbb{N}$. Then $J_{\gamma}(E) \cap I_{\gamma}^{p}(E)$ is dense in $J_{\gamma}(\bar{E})$.

For an outer function $F$ and a measurable set $\Gamma \subseteq \mathbb{T}$, let

$$
F_{\Gamma}(z)=\exp \left(\frac{1}{2 \pi} \int_{\Gamma} \frac{e^{i \theta}+z}{e^{i \theta}-z} \log \left|F\left(e^{i \theta}\right)\right| d \theta\right) \quad(z \in \mathbb{D}) .
$$

Observe that $\left|F_{\Gamma}\right|=|F|$ a.e. on $\Gamma$ and $\left|F_{\Gamma}\right|=1$ a.e. on $\mathbb{T} \backslash \Gamma$. Also, $F_{\Gamma} \rightarrow 1$ pointwise on $\mathbb{D}$ as $m(\Gamma) \rightarrow 0$. The following proof is inspired by [13].

Proof of Proposition 5.2. Let $f \in J_{\gamma}(E) \cap I_{\gamma}^{p}(E)$ and write $\mathbb{T} \backslash E=$ $\bigcup_{n=1}^{\infty} V_{n}$, where $\left(V_{n}\right)$ is a sequence of pairwise disjoint, open arcs on $\mathbb{T}$ with endpoints $a_{n}$ and $b_{n}$. For $N \in \mathbb{N}$, let $\Gamma_{N}=\bigcup_{n=N+1}^{\infty} V_{n}$ and let $F_{N}=F_{\Gamma_{N}}$. We shall prove that

(i) $F_{N} f \rightarrow f$ in $\Lambda_{\gamma}^{+}$as $N \rightarrow \infty$.

(ii) $F_{N} f \in \overline{\Lambda_{\gamma}^{+} F}$ for $N \in \mathbb{N}$.

(i): We have

$$
\left(F_{N} f-f\right)^{\prime}=\left(F_{N}-1\right) f^{\prime}+F_{N}^{\prime} f .
$$

Also, $F_{N} \rightarrow 1$ uniformly on compact subsets of $\overline{\mathbb{D}} \backslash E$, so $F_{N} f \rightarrow f$ uniformly on $\overline{\mathbb{D}}$ and

$$
\sup _{z \in \mathbb{D}}\left|\left(F_{N}(z)-1\right) f^{\prime}(z)\right|(1-|z|)^{1-\gamma} \rightarrow 0
$$

as $N \rightarrow \infty$. We shall now prove that

$$
\left|F_{N}^{\prime}(z) f(z)\right|=o\left((1-|z|)^{\gamma-1}\right)
$$

as $d(z, E) \rightarrow 0$ uniformly in $N$. For $N \in \mathbb{N}$, let $E_{N}=E \cap \bar{\Gamma}_{N}=\partial \Gamma_{N}$ and let

$$
\begin{aligned}
& G_{1 N}=\left\{z=r e^{i t} \in \mathbb{D}: d\left(e^{i t}, E_{N}\right) \leq(1-r)^{1 / 2}\right\}, \\
& G_{2 N}=\left\{z=r e^{i t} \in \mathbb{D}: d\left(e^{i t}, E_{N}\right)>(1-r)^{1 / 2} \text { and } e^{i t} \notin \Gamma_{N}\right\}, \\
& G_{3 N}=\left\{z=r e^{i t} \in \mathbb{D}: d\left(e^{i t}, E_{N}\right)>(1-r)^{1 / 2} \text { and } e^{i t} \in \Gamma_{N}\right\} .
\end{aligned}
$$

For $z=r e^{i t} \in G_{1 N}$, choose $e^{i \theta} \in E_{N}$ such that

$$
\begin{aligned}
d\left(z, E_{N}\right)^{2} & =\left|z-e^{i \theta}\right|^{2}=(1-r)^{2}+4 r \sin ^{2}(\theta-t) / 2 \\
& =(1-r)^{2}+r d\left(e^{i t}, E_{N}\right)^{2} \leq 1-r .
\end{aligned}
$$

By Cauchy's inequalities, $\left|F_{N}^{\prime}(z)\right| \leq C(1-r)^{-1}$, so

$$
\left|F_{N}^{\prime}(z) f(z)\right| \leq C d(z, E)^{p}(1-r)^{-1} \leq C(1-r)^{p / 2-1} .
$$


For $z=r e^{i t} \in G_{2 N}$, we have $d\left(e^{i t}, \Gamma_{N}\right)=d\left(e^{i t}, E_{N}\right)$ and thus

$$
d\left(z, \Gamma_{N}\right)^{2}=(1-r)^{2}+r d\left(e^{i t}, \Gamma_{N}\right)^{2}=d\left(z, E_{N}\right)^{2} .
$$

Moreover,

$$
F_{N}^{\prime}(z)=\frac{1}{\pi} \int_{\Gamma_{N}} \frac{e^{i \theta}}{\left(e^{i \theta}-z\right)^{2}} \log \left|F\left(e^{i \theta}\right)\right| d \theta \cdot F_{N}(z),
$$

so

$$
\left|F_{N}^{\prime}(z)\right| \leq C \int_{\mathbb{T}}|\log | F\left(e^{i \theta}\right)|| d \theta \cdot d\left(z, E_{N}\right)^{-2}
$$

and thus

$$
\left|F_{N}^{\prime}(z) f(z)\right| \leq C d\left(z, E_{N}\right)^{p-2} .
$$

Also, $d\left(z, E_{N}\right)^{2}=(1-r)^{2}+r d\left(e^{i t}, E_{N}\right)^{2} \geq 1-r$, so

$$
\left|F_{N}^{\prime}(z) f(z)\right| \leq C d(z, E)^{p-2 \gamma}(1-r)^{\gamma-1} .
$$

Now, let $z=r e^{i t} \in G_{3 N}$. We have

$$
F_{N}^{\prime}(z)=\frac{F^{\prime}(z) F_{N}(z)}{F(z)}-\frac{1}{\pi} \int_{\mathbb{T} \backslash \Gamma_{N}} \frac{e^{i \theta}}{\left(e^{i \theta}-z\right)^{2}} \log \left|F\left(e^{i \theta}\right)\right| d \theta \cdot F_{N}(z) .
$$

Since $d\left(z, \mathbb{T} \backslash \Gamma_{N}\right) \geq d\left(z, E_{N}\right)$, the second term can be estimated as for $z \in G_{2 N}$. For the first term, we apply [13, Lemma 1] with $\Gamma=\mathbb{T} \backslash \Gamma_{N}$ and $\eta=1 / 2$ and obtain $\left|F_{N}(z) / F(z)\right| \leq C$. Since $F \in J_{\gamma}(E)$, we have verified (10).

For $\delta>0$, let $E_{\delta}=\{z \in \mathbb{T}: d(z, E)<\delta\}$ and $U_{\delta}=\{z \in \mathbb{D}: d(z, E)<\delta\}$. Given $\varepsilon>0$, it follows from (10) that there exists $\delta>0$ such that

$$
\left|F_{N}^{\prime}(z) f(z)\right|(1-|z|)^{1-\gamma} \leq \varepsilon
$$

for $z \in U_{\delta}$ and $N \in \mathbb{N}$. Since $\bar{V}_{n} \cap E \neq \emptyset(n \in \mathbb{N})$, there exists $N_{0} \in \mathbb{N}$ such that $V_{n} \subseteq E_{\delta / 2}$ for $n>N_{0}$ and thus $\Gamma_{N} \subseteq E_{\delta / 2}$ for $N \geq N_{0}$. Hence $d\left(z, \Gamma_{N}\right) \geq \delta / 2$ for $z \notin U_{\delta}$ and $N \geq N_{0}$, so

$$
\left|F_{N}^{\prime}(z)\right| \leq C d\left(z, \Gamma_{N}\right)^{-2} \int_{\Gamma_{N}}|\log | F\left(e^{i \theta}\right)|| d \theta \rightarrow 0
$$

uniformly on $\mathbb{D} \backslash U_{\delta}$ as $N \rightarrow \infty$. We thus conclude that $F_{N} f \in \Lambda_{\gamma}^{+}$and that $F_{N} f \rightarrow f$ in $\Lambda_{\gamma}^{+}$as $N \rightarrow \infty$.

(ii): Fix $N \in \mathbb{N}$. Since $f \in J_{\gamma}(E)$, it follows from (10) that $F_{N} f \in J_{\gamma}(E)$. For $a \in \mathbb{T}$ and $\mu>0$, let

$$
K_{a \mu}(z)=\frac{a-z}{(1+\mu) a-z} \quad(z \in \overline{\mathbb{D}}) .
$$

(This is a generalization of the sequence $\left(K_{n}\right)$ introduced in Section 3.) With

$$
\Phi_{\mu}=\left(\prod_{n=1}^{N} K_{a_{n} \mu} K_{b_{n} \mu}\right)^{p}
$$


it follows from Lemma 3.3 that

$$
\Phi_{\mu} F_{N} f \rightarrow F_{N} f
$$

in $\Lambda_{\gamma}^{+}$as $\mu \rightarrow 0$. Now, fix $\mu>0$. For $\varepsilon>0$ and $n=1, \ldots, N$, let $V_{n \varepsilon}$ be the subarc of $V_{n}$ whose endpoints $c_{n}$ and $d_{n}$ are at a distance $\varepsilon$ from $a_{n}$ and $b_{n}$ respectively. Let $D_{\varepsilon}=\bigcup_{n=1}^{N} V_{n \varepsilon}$ and let

$$
\Phi_{\mu \varepsilon}=\left(\prod_{n=1}^{N} K_{c_{n} \mu} K_{d_{n} \mu}\right)^{p} .
$$

We shall show that

(a) $\Phi_{\mu \varepsilon} F_{D_{\varepsilon}}^{-1} \in \Lambda_{\gamma}^{+}$for $\varepsilon>0$

(b) $\Phi_{\mu \varepsilon} F_{D_{\varepsilon}}^{-1} F f \rightarrow \Phi_{\mu} F_{N} f$ in $\Lambda_{\gamma}^{+}$as $\varepsilon \rightarrow 0$.

It then follows from (11) that $F_{N} f \in \overline{\Lambda_{\gamma}^{+} F}$. For simplicity, we only prove (a) and (b) for $N=1$, but the proof is essentially the same in the general case.

(a): Let $\varepsilon>0$. It follows from the proof of (10) that

$$
\left|\Phi_{\mu \varepsilon}(z) F_{D_{\varepsilon}}^{\prime}(z)\right| \leq C(1-|z|)^{\gamma-1} \quad(z \in \mathbb{D}) .
$$

Also, the outer function $F_{D_{\varepsilon}}$ is bounded away from zero on $\mathbb{T}$ and thus on $\overline{\mathbb{D}}$, so

$$
\left|\Phi_{\mu \varepsilon}(z)\left(F_{D_{\varepsilon}}^{-1}\right)^{\prime}(z)\right| \leq C(1-|z|)^{\gamma-1} \quad(z \in \mathbb{D}),
$$

and (a) follows.

(b): For $\varepsilon>0$, let $W_{\varepsilon}=V_{1} \backslash V_{1 \varepsilon}$ so that $\partial W_{\varepsilon}=\left\{a_{1}, c_{1}, d_{1}, b_{1}\right\}$. Then

$$
F_{V_{1 \varepsilon}}^{-1} F=F_{W_{\varepsilon}} F_{1} \text {, }
$$

so

$$
\begin{aligned}
\left(\Phi_{\mu \varepsilon} F_{V_{1 \varepsilon}}^{-1} F f-\Phi_{\mu} F_{1} f\right)^{\prime} & \\
= & \left(\Phi_{\mu \varepsilon} F_{W_{\varepsilon}} F_{1}-\Phi_{\mu} F_{1}\right) f^{\prime}+\left(\Phi_{\mu \varepsilon} F_{W_{\varepsilon}}-\Phi_{\mu}\right) F_{1}^{\prime} f \\
& +\left(\Phi_{\mu \varepsilon}^{\prime} F_{W_{\varepsilon}}-\Phi_{\mu}^{\prime}\right) F_{1} f+\Phi_{\mu \varepsilon} F_{W_{\varepsilon}}^{\prime} F_{1} f .
\end{aligned}
$$

As $\varepsilon \rightarrow 0$, we have $\Phi_{\mu \varepsilon} \rightarrow \Phi_{\mu}$ and $\Phi_{\mu \varepsilon}^{\prime} \rightarrow \Phi_{\mu}^{\prime}$ uniformly on $\overline{\mathbb{D}}$ and $F_{W_{\varepsilon}} \rightarrow 1$ uniformly on compact subsets of $\overline{\mathbb{D}} \backslash\left\{a_{1}, b_{1}\right\}$, so

$$
\begin{aligned}
\sup _{z \in \mathbb{D}} \mid\left[\left(\Phi_{\mu \varepsilon} F_{W_{\varepsilon}} F_{1}-\Phi_{\mu} F_{1}\right) f^{\prime}\right. & +\left(\Phi_{\mu \varepsilon} F_{W_{\varepsilon}}-\Phi_{\mu}\right) F_{1}^{\prime} f \\
& \left.+\left(\Phi_{\mu \varepsilon}^{\prime} F_{W_{\varepsilon}}-\Phi_{\mu}^{\prime}\right) F_{1} f\right](z) \mid \cdot(1-|z|)^{1-\gamma} \rightarrow 0 .
\end{aligned}
$$

In order to estimate the last term on the right-hand side of (12), we shall imitate the proof of (10). For $\varepsilon>0$, let $f_{\varepsilon}=\Phi_{\mu \varepsilon} f \in I_{\gamma}^{p}\left(\partial W_{\varepsilon}\right)$ and let

$$
\begin{aligned}
& G_{1 \varepsilon}=\left\{z=r e^{i t} \in \mathbb{D}: d\left(e^{i t}, \partial W_{\varepsilon}\right) \leq(1-r)^{1 / 2}\right\}, \\
& G_{2 \varepsilon}=\left\{z=r e^{i t} \in \mathbb{D}: d\left(e^{i t}, \partial W_{\varepsilon}\right)>(1-r)^{1 / 2} \text { and } e^{i t} \notin W_{\varepsilon}\right\}, \\
& G_{3 \varepsilon}=\left\{z=r e^{i t} \in \mathbb{D}: d\left(e^{i t}, \partial W_{\varepsilon}\right)>(1-r)^{1 / 2} \text { and } e^{i t} \in W_{\varepsilon}\right\} .
\end{aligned}
$$


For $z=r e^{i t} \in G_{1 \varepsilon}$, we have $d\left(z, \partial W_{\varepsilon}\right)^{2} \leq 1-r$, so

$$
\left|F_{W_{\varepsilon}}^{\prime}(z) f_{\varepsilon}(z)\right| \leq C(1-r)^{p / 2-1}
$$

uniformly for $\varepsilon>0$. Moreover, $F_{W_{\varepsilon}}^{\prime} \rightarrow 0$ uniformly on compact subsets of $\mathbb{D}$, so

$$
\sup _{z \in G_{1 \varepsilon}}\left|F_{W_{\varepsilon}}^{\prime}(z) f_{\varepsilon}(z)\right|(1-|z|)^{1-\gamma} \rightarrow 0
$$

as $\varepsilon \rightarrow 0$. Also, for $z=r e^{i t} \in G_{2 \varepsilon}$, we have

$$
\left|F_{W_{\varepsilon}}^{\prime}(z) f_{\varepsilon}(z)\right|(1-r)^{1-\gamma} \leq C \int_{W_{\varepsilon}}|\log | F\left(e^{i \theta}\right)|| d \theta \rightarrow 0
$$

as $\varepsilon \rightarrow 0$. For $z=r e^{i t} \in G_{3 \varepsilon}$, we have $d\left(z, \partial W_{\varepsilon}\right)^{2} \leq 2 d\left(e^{i t}, \partial W_{\varepsilon}\right)^{2} \leq 2 \varepsilon^{2}$. Moreover,

$$
F_{W_{\varepsilon}}^{\prime}(z)=\frac{F^{\prime}(z) F_{W_{\varepsilon}}(z)}{F(z)}-\frac{1}{\pi} \int_{\mathbb{T} \backslash W_{\varepsilon}} \frac{e^{i \theta}}{\left(e^{i \theta}-z\right)^{2}} \log \left|F\left(e^{i \theta}\right)\right| d \theta \cdot F_{W_{\varepsilon}}(z),
$$

and $\left|F_{W_{\varepsilon}}(z) / F(z)\right| \leq C$ by [13, Lemma 1], so

$$
\begin{aligned}
\left|F_{W_{\varepsilon}}^{\prime}(z) f_{\varepsilon}(z)\right| & (1-r)^{1-\gamma} \\
\leq & C d\left(z, \partial W_{\varepsilon}\right)^{p}\left((1-r)^{\gamma-1}+d\left(z, \partial W_{\varepsilon}\right)^{-2}\right)(1-r)^{1-\gamma} \\
\leq & \leq\left(d\left(z, \partial W_{\varepsilon}\right)^{p}+d\left(z, \partial W_{\varepsilon}\right)^{p-2+2(1-\gamma)}\right) \leq C\left(\varepsilon^{p}+\varepsilon^{p-2 \gamma}\right) .
\end{aligned}
$$

All in all, we conclude that

$$
\sup _{z \in \mathbb{D}}\left|F_{W_{\varepsilon}}^{\prime}(z) f_{\varepsilon}(z)\right|(1-|z|)^{1-\gamma} \rightarrow 0
$$

as $\varepsilon \rightarrow 0$, so (b) follows from (12).

We now turn to the proof of Proposition 5.3. In the proof of the corresponding result for $\lambda_{\gamma}^{+}([12$, Theorem A $])$, the first step is that if $f \in \lambda_{\gamma}^{+}$ with $f=F Q$, where $F$ is an outer and $Q$ an inner function, then

$$
f_{t}=F^{1+t} Q \rightarrow f
$$

in $\lambda_{\gamma}^{+}$as $t \rightarrow 0$, and moreover $f_{t} \in I_{\gamma}^{(1+t) \gamma}(Z(F))$. In our case, for $f \in J_{\gamma}(E)$, we only have $f_{t} \rightarrow f$ in $\Lambda_{\gamma}^{+}$as $t \rightarrow 0$ if $Z(F)=E$, and this complicates the proof of Proposition 5.3. We shall need the following factorization result, which we find interesting in itself.

Proposition 5.4. Let $F \in \Lambda_{\gamma}^{+}$be an outer function and suppose that $Z(F)=E_{1} \cup E_{2}$, where $E_{1}, E_{2} \subseteq \mathbb{T}$ are closed, disjoint sets. Then there exist outer functions $F_{1}, F_{2} \in \Lambda_{\gamma}^{+}$such that $F=F_{1} F_{2}$ and $Z\left(F_{k}\right)=E_{k}(k=1,2)$.

Proof. Choose open sets $U_{1}, U_{2}, V_{1}, V_{2} \subseteq \mathbb{T}$ such that $E_{k} \subseteq U_{k}, \bar{U}_{k} \subseteq V_{k}$ $(k=1,2)$ and such that $V_{1}$ and $V_{2}$ are disjoint, and choose $\chi_{1}, \chi_{2} \in \Lambda_{\gamma}$ 
such that $\chi_{1}+\chi_{2}=1$ on $\mathbb{T}$ and $\chi_{k}=1$ on $U_{k}(k=1,2)$. For $k=1,2$, let $\varphi_{k}=\chi_{k} \log |F|$ and define an outer function $F_{k}$ by

$$
F_{k}(z)=\exp \left(\frac{1}{2 \pi} \int_{\mathbb{T}} \frac{e^{i \theta}+z}{e^{i \theta}-z} \varphi_{k}\left(e^{i \theta}\right) d \theta\right) \quad(z \in \mathbb{D}) .
$$

Then $Z\left(F_{k}\right)=E_{k}$ and $F=F_{1} F_{2}$. Choose $\psi_{k} \in \Lambda_{\gamma}$ such that $\psi_{k}=\varphi_{k}$ on $\mathbb{T} \backslash U_{k}$ and let

$$
\begin{aligned}
& G_{k}(z)=\exp \left(\frac{1}{2 \pi} \int_{\mathbb{T}} \frac{e^{i \theta}+z}{e^{i \theta}-z} \psi_{k}\left(e^{i \theta}\right) d \theta\right), \\
& H_{k}(z)=\exp \left(\frac{1}{2 \pi} \int_{\mathbb{T}} \frac{e^{i \theta}+z}{e^{i \theta}-z}\left(\varphi_{k}\left(e^{i \theta}\right)-\psi_{k}\left(e^{i \theta}\right)\right) d \theta\right)
\end{aligned}
$$

for $z \in \mathbb{D}$, so that $F_{k}=G_{k} H_{k}$. Since $\Lambda_{\gamma}$ is closed under harmonic conjugation ([17, Theorem III.13.29]), it follows that $\log G_{k} \in \Lambda_{\gamma}^{+}$and thus $G_{k}, G_{k}^{-1} \in \Lambda_{\gamma}^{+}$. For $e^{i \theta} \in U_{1}$, the function $z \mapsto\left(e^{i \theta}+z\right) /\left(e^{i \theta}-z\right)$ belongs to $\Lambda_{\gamma}\left(\mathbb{T} \backslash V_{1}\right)$, so we deduce that $H_{1} \in \Lambda_{\gamma}\left(\mathbb{T} \backslash V_{1}\right)$ and thus $F_{1} \in \Lambda_{\gamma}\left(\mathbb{T} \backslash V_{1}\right)$. Similarly $F_{2} \in \Lambda_{\gamma}\left(\mathbb{T} \backslash V_{2}\right)$, so $F_{1}=F / F_{2} \in \Lambda_{\gamma}\left(\mathbb{T} \backslash V_{2}\right)$ since $F_{2}$ has no zeros on $\mathbb{T} \backslash V_{2}$. Hence $F_{1} \in \Lambda_{\gamma}$ and thus $F_{1} \in \Lambda_{\gamma}^{+}$. Similarly $F_{2} \in \Lambda_{\gamma}^{+}$.

Proof of Proposition 5.3. Let $f \in J_{\gamma}(E)$ with $f=F Q$, where $F$ is an outer and $Q$ an inner function, and let $\varepsilon>0$. Choose $0<\delta \leq \varepsilon$ such that

$$
\left|f^{\prime}(z)\right|<\varepsilon(1-|z|)^{\gamma-1}
$$

for $z \in U_{\delta}$, where $U_{\delta}$ and $E_{\delta}$ are as in the proof of Proposition 5.2. It is easily seen that there exist closed, disjoint sets $E_{1}, E_{2} \subseteq \mathbb{T}$ with $E \subseteq E_{1} \subseteq E_{\delta}$ and $Z(F)=E_{1} \cup E_{2}$, so it follows from the previous proposition that $F=F_{1} F_{2}$, where $F_{1}, F_{2} \in \Lambda_{\gamma}^{+}$are outer functions with $Z\left(F_{k}\right)=E_{k}(k=1,2)$. For $t>0$, let

$$
f_{t}=F_{1}^{1+t} F_{2} Q=F_{1}^{t} f
$$

so that

$$
f_{t}^{\prime}=t F_{1}^{t-1} F_{1}^{\prime} f+F_{1}^{t} f^{\prime}=F_{1}^{t}\left(t F_{1}^{\prime} F_{2} Q+f^{\prime}\right) .
$$

Since $F_{1}=0$ on $E_{1} \supseteq E$, we deduce that $f_{t} \in J_{\gamma}(E) \cap I_{\gamma}^{(1+t) \gamma}(E)$. Moreover,

$$
\left(f_{t}-f\right)^{\prime}=t F_{1}^{t} F_{1}^{\prime} F_{2} Q+\left(F_{1}^{t}-1\right) f^{\prime} .
$$

Since $Z\left(F_{1}\right) \subseteq E_{\delta}$, we have $F_{1}^{t} \rightarrow 1$ uniformly on $\mathbb{D} \backslash U_{\delta}$ as $t \rightarrow 0$, so

$$
\limsup _{t \rightarrow 0}\left\|f_{t}-f\right\|_{\Lambda_{\gamma}^{+}} \leq C \sup _{z \in U_{\delta}}\left|f^{\prime}(z)\right|(1-|z|)^{1-\gamma}<C \varepsilon .
$$

Write $\mathbb{T} \backslash E_{1}=\bigcup_{n=1}^{\infty} W_{n}$, where $\left(W_{n}\right)$ is a sequence of pairwise disjoint, open $\operatorname{arcs}$ on $\mathbb{T}$. For $N \in \mathbb{N}$, let $\Omega_{N}=\bigcup_{n=N+1}^{\infty} W_{n}$ and let

$$
F_{1 N}=\left(F_{1}\right)_{\Omega_{N}}
$$


(see (9)). Fix $t>0$ and let $q \in \mathbb{N}$. We have $F_{1 N}^{q} \rightarrow 1$ uniformly on compact subsets of $\overline{\mathbb{D}} \backslash E_{1}$ and $f_{t}=0$ on $E_{1}$, so $F_{1 N}^{q} f_{t} \rightarrow f_{t}$ uniformly on $\overline{\mathbb{D}}$. To estimate $\left(F_{1 N}^{q}\right)^{\prime} f_{t}=q F_{1 N}^{q-1} F_{1 N}^{\prime} f_{t}$ on $\mathbb{D} \backslash U_{\delta}$, we choose $N_{0} \in \mathbb{N}$ such that $\Omega_{N} \subseteq E_{\delta / 2}$ for $N \geq N_{0}$. We have

$$
\left|F_{1 N}^{\prime}(z)\right| \leq C d\left(z, \Omega_{N}\right)^{-2} \int_{\Omega_{N}}|\log | F\left(e^{i \theta}\right)|| d \theta \leq C \delta^{-2} \int_{\Omega_{N}}|\log | F\left(e^{i \theta}\right)|| d \theta \rightarrow 0
$$

uniformly for $z \in \mathbb{D} \backslash U_{\delta}$ as $N \rightarrow \infty$. To estimate $\left(F_{1 N}^{q}\right)^{\prime} f_{t}$ on $U_{\delta}$, we repeat the proof of [12, Theorem B] (for $q$ sufficiently large) with $d(z)=d\left(z, E_{1}\right)$ and use the fact that

$$
|f(z)| \leq C\left|F_{1}(z)\right| \leq C d\left(z, E_{1}\right)^{\gamma} \leq C \varepsilon^{\gamma},
$$

and obtain

$$
\limsup _{N \rightarrow \infty} \sup _{z \in U_{\delta}}\left|\left(F_{1 N}^{q}\right)^{\prime}(z) f_{t}(z)\right|(1-|z|)^{1-\gamma}=\kappa(\varepsilon),
$$

where $\kappa(\varepsilon) \rightarrow 0$ as $\varepsilon \rightarrow 0$. Moreover, by (13), we have

$$
\sup _{z \in U_{\delta}}\left|f_{t}^{\prime}(z)\right|(1-|z|)^{1-\gamma} \leq C \sup _{z \in U_{\delta}}\left|F_{1}^{t}(z)\right| \leq C \delta^{t \gamma} \leq C \varepsilon^{t \gamma},
$$

so

$$
\limsup _{N \rightarrow \infty}\left\|f_{t}-F_{1 N}^{q} f_{t}\right\|_{\Lambda_{\gamma}^{+}}=\widetilde{\kappa}(\varepsilon)
$$

where $\widetilde{\kappa}(\varepsilon) \rightarrow 0$ as $\varepsilon \rightarrow 0$.

Now, fix $N \in \mathbb{N}$. It follows from the above that $F_{1 N}^{q} f_{t} \in J_{\gamma}(E)$. Moreover,

$$
\left|F_{1 N}^{q}(z)\right| \leq C d\left(z, \partial \Omega_{N}\right)^{p} \quad(z \in \overline{\mathbb{D}})
$$

for $q \geq p / \gamma$. Since $\partial\left(\mathbb{T} \backslash E_{1}\right)=E_{1}$, we deduce that $E \backslash \partial \Omega_{N}$ is finite, say $E \backslash \partial \Omega_{N}=\left\{a_{1}, \ldots, a_{M}\right\}$. By Lemma 3.3, we then have

$$
\left(\prod_{m=1}^{M} K_{a_{m} \mu}\right)^{p} F_{1 N}^{q} f_{t} \rightarrow F_{1 N}^{q} f_{t}
$$

in $\Lambda_{\gamma}^{+}$as $\mu \rightarrow 0$, and since

$$
\left(\prod_{m=1}^{M} K_{a_{m} \mu}\right)^{p} F_{1 N}^{q} \in J_{\gamma}(E) \cap I_{\gamma}^{p}(E),
$$

this finishes the proof.

6. Weak-star closed ideals. In this section, we characterize the $\mathrm{wk}^{*}$ closed ideals in $\Lambda_{\gamma}^{+}$. We begin by describing the wk* topology on $\Lambda_{\gamma}$ and $\Lambda_{\gamma}^{+}$. For $z \in \mathbb{T}$, let $\delta_{z} \in \Lambda_{\gamma}^{*}$ be the point evaluation functional at $z$, and let

$$
Y_{\gamma}=\overline{\operatorname{span}\left\{\delta_{z}: z \in \mathbb{T}\right\}}
$$


(norm closure in $\left.\Lambda_{\gamma}^{*}\right)$. Johnson ([7, Section 4]) proved that

$$
Y_{\gamma}^{*}=\Lambda_{\gamma} \text {. }
$$

Moreover, a bounded net in $\Lambda_{\gamma}$ converges wk* to zero in $\Lambda_{\gamma}$ if and only if it converges pointwise to zero on $\mathbb{T}$, and in this case it actually converges uniformly to zero on $\mathbb{T}$. When $0<\gamma<1$, we further have $Y_{\gamma}=\lambda_{\gamma}^{*}$ and thus $\Lambda_{\gamma}=\lambda_{\gamma}^{* *}([7$, Theorem 4.7]).

Lemma 6.1. Multiplication is separately $w k^{*}$ continuous in $\Lambda_{\gamma}$.

Proof. The space $Y_{\gamma}^{* *}=\Lambda_{\gamma}^{*}$ is a Banach $\Lambda_{\gamma}$-module under the action

$$
\langle f, g \varphi\rangle=\langle f g, \varphi\rangle \quad\left(f, g \in \Lambda_{\gamma}, \varphi \in Y_{\gamma}^{* *}\right) .
$$

For $z \in \mathbb{T}$ and $g \in \Lambda_{\gamma}$, we have

$$
\left\langle f, g \delta_{z}\right\rangle=f(z) g(z) \quad\left(f \in \Lambda_{\gamma}\right),
$$

so $g \delta_{z}=g(z) \delta_{z}$. Hence $Y_{\gamma}$ is a $\Lambda_{\gamma}$-submodule and the conclusion follows.

Let $\left(f_{n}\right)$ be a sequence in $\Lambda_{\gamma}^{+}$which converges wk* to $f$ in $\Lambda_{\gamma}$ as $n \rightarrow \infty$. Then $\widehat{f}_{n}(m) \rightarrow \widehat{f}(m)$ as $n \rightarrow \infty$ for $m \in \mathbb{Z}$ by Lebesgue's dominated convergence theorem. Hence $f \in \Lambda_{\gamma}^{+}$, so $\Lambda_{\gamma}^{+}$is wk* closed by the Krein-Šmulian theorem. Denoting the quotient space $Y_{\gamma} /^{\perp}\left(\Lambda_{\gamma}^{+}\right)$by $Y_{\gamma}^{+}$, we thus have

$$
\Lambda_{\gamma}^{+}=\left(Y_{\gamma}^{+}\right)^{*} \text {. }
$$

The next result often provides us with the easiest way to show wk* convergence in $\Lambda_{\gamma}^{+}$.

Lemma 6.2. Let $\left(f_{n}\right)$ be a bounded sequence in $\Lambda_{\gamma}^{+}$which converges pointwise to zero on $\mathbb{D}$ as $n \rightarrow \infty$. Then $f_{n} \rightarrow 0 w k^{*}$ in $\Lambda_{\gamma}^{+}$as $n \rightarrow \infty$.

Proof. Let $z \in \mathbb{T}$ and $\varepsilon>0$. Choose $w \in \mathbb{D}$ with $|z-w|<\varepsilon$. Since $f_{n}(w) \rightarrow 0$ as $n \rightarrow \infty$ and since $\left(f_{n}\right)$ is bounded in $\Lambda_{\gamma}^{+}$, it follows that $\lim \sup _{n \rightarrow \infty}\left|f_{n}(z)\right| \leq C \varepsilon^{\gamma}$. Hence $f_{n} \rightarrow 0$ pointwise on $\mathbb{T}$ as $n \rightarrow \infty$ and the result follows.

We now turn our attention to $\mathrm{wk}^{*}$ closed ideals in $\Lambda_{\gamma}^{+}$.

Proposition 6.3. Suppose that a closed set $E \subseteq \mathbb{T}$ and an inner function $Q$ satisfy (2). Then $I_{\gamma}(E, Q)$ is a wk* closed ideal in $\Lambda_{\gamma}^{+}$.

Proof. Let $\left(f_{n}\right)$ be a sequence in $I_{\gamma}(E, Q)$ and suppose that $f_{n} \rightarrow f$ wk $^{*}$ in $\Lambda_{\gamma}^{+}$as $n \rightarrow \infty$ for some $f \in \Lambda_{\gamma}^{+}$. Then $f \in I_{\gamma}(E)$ and it follows from Theorem 1.1 that $\left(f_{n} / Q\right)$ is a bounded sequence in $\Lambda_{\gamma}^{+}$. Moreover, $f_{n} / Q \rightarrow f / Q$ pointwise on $\mathbb{T}$ as $n \rightarrow \infty$, so we deduce that $f_{n} / Q \rightarrow f / Q$ wk $^{*}$ in $\Lambda_{\gamma}^{+}$as $n \rightarrow \infty$. In particular, $f \in I_{\gamma}(E, Q)$. The Krein-Šmulian theorem thus implies that $I_{\gamma}(E, Q)$ is wk* closed.

The aim of this section is to prove the following result, which states that the ideals $I_{\gamma}(E, Q)$ are the only $\mathrm{wk}^{*}$ closed ideals in $\Lambda_{\gamma}^{+}$. 
Theorem 6.4. Let I be a $w k^{*}$ closed ideal in $\Lambda_{\gamma}^{+}$. Then

$$
I=I_{\gamma}\left(E_{I}, Q_{I}\right) \text {. }
$$

The proof of the theorem takes up the rest of this paper. The idea in the proof is similar to that of [10] and [11]. Firstly, the Carleman transform is used to show that a wk* closed ideal $I$ in $\Lambda_{\gamma}^{+}$with $Q_{I}=1$ necessarily contains a certain class of functions. Secondly, we show that every function in $I_{\gamma}(E, Q)$ can be approximated by sufficiently smooth functions. Finally, the result is deduced from these two facts.

For a $\left(\mathrm{wk}^{*}\right)$ closed ideal $I$ in $\Lambda_{\gamma}^{+}$, we let

$$
{ }^{\perp} I=\left\{\varphi \in Y_{\gamma}^{+}:\langle\varphi, f\rangle=0 \text { for every } f \in I\right\}=I^{\perp} \cap Y_{\gamma}^{+} .
$$

Also, for an inner function $Q$, a closed set $Z \subseteq \overline{\mathbb{D}}$ and $p>0$, let

$$
I_{\gamma}^{p}(Z, Q)=\left\{f \in \Lambda_{\gamma}^{+}: f / Q \in \Lambda_{\gamma}^{+} \text {and }|f(z)| \leq C d(z, Z)^{p}(z \in \mathbb{T})\right\},
$$

so that $I_{\gamma}^{p}(E)=I_{\gamma}^{p}(E, 1)$ for a closed set $E \subseteq \mathbb{T}$ (see the previous section). For $f \in \Lambda_{\gamma}^{+}$, we have $\left\|f_{r}\right\|_{\Lambda_{\gamma}^{+}} \leq\|f\|_{\Lambda_{\gamma}^{+}}$for $r<1$ and thus $f_{r} \rightarrow f \mathrm{wk}^{*}$ in $\Lambda_{\gamma}^{+}$ as $r \rightarrow 1_{-}$, so we can use a method from [10] in the proof of the next result.

Lemma 6.5. Let I be a $w k^{*}$ closed ideal in $\Lambda_{\gamma}^{+}$with $Q_{I}=1$. Then

$$
I_{\gamma}^{2(1+\gamma)}\left(E_{I}, 1\right) \subseteq I .
$$

Proof. Let $f \in I_{\gamma}^{2(1+\gamma)}\left(E_{I}, 1\right)$ and suppose that $\varphi \in^{\perp} I$. Then

$$
\langle\varphi, f\rangle=\lim _{r \rightarrow 1_{-}}\left\langle\varphi, f_{r}\right\rangle=\lim _{s \rightarrow 1_{+}} \frac{1}{2 \pi} \int_{\mathbb{T}} f\left(e^{i \theta}\right) e^{i \theta} \Phi\left(s e^{i \theta}\right) d \theta .
$$

From the proof of [10, Lemma 3.3] (see also [11, Theorem 5]), we deduce that

$$
|\Phi(z)| \leq C d\left(z, E_{I}\right)^{-2(1+\gamma)} \quad(z \in \mathbb{C} \backslash \overline{\mathbb{D}}),
$$

so it follows from Lebesgue's dominated convergence theorem that

$$
\langle\varphi, f\rangle=\frac{1}{2 \pi} \int_{\mathbb{T}} f\left(e^{i \theta}\right) e^{i \theta} \Phi\left(e^{i \theta}\right) d \theta .
$$

By the Beurling-Rudin theorem, the space $I$ is dense in the Hardy space $\mathcal{H}^{2}$, so there exists a sequence $\left(f_{n}\right)$ in $I$ converging to 1 in $\mathcal{H}^{2}$. Since $f f_{n} \in I$, we thus have

$$
\langle\varphi, f\rangle=\lim _{n \rightarrow \infty} \frac{1}{2 \pi} \int_{\mathbb{T}} f\left(e^{i \theta}\right) f_{n}\left(e^{i \theta}\right) e^{i \theta} \Phi\left(e^{i \theta}\right) d \theta=\lim _{n \rightarrow \infty}\left\langle\varphi, f f_{n}\right\rangle=0 .
$$

Hence $f \in I$ by the Hahn-Banach theorem.

The main difficulty in the proof of Theorem 6.4 is contained in the following approximation result. 
Proposition 6.6. Let $p>0$ and suppose that a closed set $E \subseteq \mathbb{T}$ and an inner function $Q$ satisfy (2). Let $Z=E \cup Z(B)$. Then $I_{\gamma}^{p}(Z, Q)$ is wk* dense in $I_{\gamma}(E, Q)$.

In order to prove the proposition, we shall need a series of lemmas. The following result should be compared with the comments before the proof of Proposition 5.4.

LEMMA 6.7. Let $f=F Q \in \Lambda_{\gamma}^{+}$, where $F$ is an outer and $Q$ an inner function. Then $f_{t}=F^{1+t} Q \in \Lambda_{\gamma}^{+}$for $t>0$ and $f_{t} \rightarrow f w k^{*}$ in $\Lambda_{\gamma}^{+}$as $t \rightarrow 0$.

Proof. We have $F \in \Lambda_{\gamma}^{+}$by Theorem 1.1. Since $f^{\prime}=F^{\prime} Q+F Q^{\prime}$, it thus follows that

$$
\sup _{z \in \mathbb{D}}\left|F(z) Q^{\prime}(z)\right|(1-|z|)^{1-\gamma}<\infty .
$$

Moreover, $f_{t}^{\prime}=(1+t) F^{t} F^{\prime} Q+F^{1+t} Q^{\prime}$, so we deduce that $\left(f_{t}\right)$ is bounded in $\Lambda_{\gamma}^{+}$as $t \rightarrow 0$. Finally, $f_{t} \rightarrow f$ pointwise on $\mathbb{T}$ as $t \rightarrow 0$, so $f_{t} \rightarrow f$ wk $^{*}$ in $\Lambda_{\gamma}^{+}$as $t \rightarrow 0$.

For $a \in \mathbb{T}$ and $\mu>0$, let $K_{a \mu}$ be as in the previous section. For $f \in \Lambda_{\gamma}^{+}$ with $f(a)=0$, it follows from the proof of Lemma 3.3 that

$$
\sup _{z \in \mathbb{D}}\left|K_{a \mu}^{\prime}(z) f(z)\right|(1-|z|)^{1-\gamma} \leq C
$$

for $\mu>0$. Hence $\left(K_{a \mu} f\right)$ is bounded in $\Lambda_{\gamma}^{+}$, and since $K_{a \mu} f \rightarrow f$ pointwise on $\mathbb{T}$, we deduce that $K_{a \mu} f \rightarrow f \mathrm{wk}^{*}$ in $\Lambda_{\gamma}^{+}$as $\mu \rightarrow 0$. From this, it is easy to deduce the following result. Then

LEMmA 6.8. Let $p \geq 1$, let $f \in \Lambda_{\gamma}^{+}$and let $\left\{a_{1}, \ldots, a_{N}\right\} \subseteq Z(f) \cap \mathbb{T}$.

$$
\left(\prod_{n=1}^{N} K_{a_{n} \mu}\right)^{p} f \rightarrow f
$$

$w k^{*}$ in $\Lambda_{\gamma}^{+}$as $\mu \rightarrow 0$.

For an outer function $F$ and a measurable set $\Gamma \subseteq \mathbb{T}$, recall the definition of $F_{\Gamma}$ from (9). From the proof of [12, Theorem B], we obtain the following result.

LEMMA 6.9. Let $F$ be an outer function, $Q$ an inner function and suppose that $F Q \in \Lambda_{\gamma}^{+}$. Let $t>0$ and let $f=F^{1+t} Q$. Then there exists $q_{0}$ such that, for $q \geq q_{0}$, we have

$$
F_{\Gamma}^{q} f \in \Lambda_{\gamma}^{+} \quad \text { with } \quad\left\|F_{\Gamma}^{q} f\right\|_{\Lambda_{\gamma}^{+}} \leq C
$$

for every open set $\Gamma \subseteq \mathbb{T}$ with $\partial \Gamma \subseteq Z(f)$ (where $\partial \Gamma$ denotes the boundary of $\Gamma$ in $\mathbb{T})$.

Proof of Proposition 6.6. By Lemma 6.7, it is sufficient to prove that, whenever a function $f \in I_{\gamma}(E, Q)$ is of the form $f=F^{1+t} Q$, where $t>0$, 
$F$ is an outer function and $Q$ an inner function such that $F Q \in \Lambda_{\gamma}^{+}$, then $f$ can be approximated in the wk* topology on $\Lambda_{\gamma}^{+}$by functions from $I_{\gamma}^{p}(Z, Q)$. Let $q=\max \left\{q_{0}, p / \gamma\right\}$. As in the proof of Proposition 5.2, let $\mathbb{T} \backslash E=\bigcup_{n=1}^{\infty} V_{n}$, where $\left(V_{n}\right)$ is a sequence of pairwise disjoint, open arcs on $\mathbb{T}$ with endpoints $a_{n}$ and $b_{n}$, and for $N \in \mathbb{N}$, let $\Gamma_{N}=\bigcup_{n=N+1}^{\infty} V_{n}$ and $F_{N}=F_{\Gamma_{N}}$. As $N \rightarrow \infty$, we have $m\left(\Gamma_{N}\right) \rightarrow 0$ and thus $F_{N} \rightarrow 1$ pointwise on $\mathbb{D}$, so it follows from Lemmas 6.2 and 6.9 that $F_{N}^{q} f \rightarrow f$ wk $^{*}$ in $\Lambda_{\gamma}^{+}$for every $q \geq q_{0}$.

Let $N \in \mathbb{N}$ be fixed. We have $E \backslash \bar{\Gamma}_{N} \subseteq\left\{a_{1}, b_{1}, \ldots, a_{N}, b_{N}\right\}$ and

$$
\left(\prod_{n=1}^{N} K_{a_{n} \mu} K_{b_{n} \mu}\right)^{p} F_{N}^{q} f \rightarrow F_{N}^{q} f
$$

$\mathrm{wk}^{*}$ in $\Lambda_{\gamma}^{+}$as $\mu \rightarrow 0$ by Lemma 6.8 ,

Fix $\mu>0$. For $\varepsilon>0$ and $n=1, \ldots, N$, let $V_{n \varepsilon}$ be the subarc of $V_{n}$ whose endpoints $c_{n}$ and $d_{n}$ are at a distance $\varepsilon$ from $a_{n}$ and $b_{n}$ respectively. Let

$$
g_{\varepsilon}=\left(\prod_{n=1}^{N} K_{a_{n} \mu} K_{c_{n} \mu} K_{d_{n} \mu} K_{b_{n} \mu}\right)^{p / 2}\left(\prod_{n=1}^{N} F_{V_{n} \backslash V_{n \varepsilon}}\right)^{q} F_{N}^{q} f .
$$

It follows from the proof of $\left[12\right.$, Theorem B] that $\left(g_{\varepsilon}\right)$ is bounded in $\Lambda_{\gamma}^{+}$as $\varepsilon \rightarrow 0$, so

$$
g_{\varepsilon} \rightarrow\left(\prod_{n=1}^{N} K_{a_{n} \mu} K_{b_{n} \mu}\right)^{p} F_{N}^{q} f
$$

$\mathrm{wk}^{*}$ in $\Lambda_{\gamma}^{+}$as $\varepsilon \rightarrow 0$ by Lemma 6.2 .

Finally, fix $\varepsilon>0$. For $z \in \bar{\Gamma}_{N}$, we have $\left|F_{N}(z)\right|=|f(z)|$, and for $z \in$ $V_{n} \backslash V_{n \varepsilon}$ for some $n \in\{1, \ldots, N\}$, we have $\left|F_{V_{n} \backslash V_{n \varepsilon}}(z)\right|=|f(z)|$. In both cases, we thus have

$$
\left|g_{\varepsilon}(z)\right| \leq C|f(z)|^{q} \leq C d(z, Z)^{p} .
$$

Clearly, this also holds for $z \in \bigcup_{n=1}^{N} \bar{V}_{n \varepsilon}$, so $g_{\varepsilon} \in I_{\gamma}^{p}(Z, Q)$, which finishes the proof.

It follows from Lemma 6.5 and Proposition 6.6 that Theorem 6.4 holds for closed ideals $I$ with $Q_{I}=1$. We now finish the proof of the general case.

Proof of Theorem 6.4. Korenblum ([9], see also [10]) has shown that there exists an outer function $T$ satisfying the following conditions:

(i) $T^{\varepsilon} Q_{I} \in \Lambda_{\gamma}^{+}$for every $\varepsilon>0$,

(ii) $Z(T)=E_{I}$,

(iii) $\left|T^{\prime}(z) / T(z)\right| \leq C d\left(z, Z_{I}\right)^{-2}(z \in \mathbb{T})$. 
Let $\varepsilon>0$ and consider the division ideal

$$
I_{\varepsilon}=\left\{f \in \Lambda_{\gamma}^{+}: T^{\varepsilon} Q_{I} f \in I\right\}
$$

in $\Lambda_{\gamma}^{+}$. Since multiplication is separately wk* continuous in $\Lambda_{\gamma}^{+}$(Lemma 6.1), it follows that $I_{\varepsilon}$ is wk* closed. Moreover, for $g \in I$, we have $g / Q_{I} \in I_{\varepsilon}$, so we deduce that $Q_{I_{\varepsilon}}=1$ and $E_{I_{\varepsilon}}=E_{I}$. As mentioned before the proof, we thus have $I_{\varepsilon}=I_{\gamma}\left(E_{I}, 1\right)$.

Now, let $g \in I_{\gamma}^{2}\left(Z_{I}, Q_{I}\right)$. Then $g / Q_{I} \in I_{\gamma}\left(E_{I}, 1\right)=I_{\varepsilon}$, so $T^{\varepsilon} g \in I$. It follows from (iii) that

$$
\left|\left(T^{\varepsilon}\right)^{\prime}(z) g(z)\right|=\left|\varepsilon T^{\varepsilon}(z)\left(T^{\prime}(z) / T(z)\right) g(z)\right| \leq C \quad(z \in \mathbb{T})
$$

for $\varepsilon>0$. Hence $T^{\varepsilon} g$ is bounded in $\Lambda_{\gamma}^{+}$as $\varepsilon \rightarrow 0$ and since $T^{\varepsilon} g \rightarrow g$ pointwise on $\mathbb{T}$ as $\varepsilon \rightarrow 0$, we have $T^{\varepsilon} g \rightarrow g$ wk* $^{*}$ in $\Lambda_{\gamma}^{+}$as $\varepsilon \rightarrow 0$, so $g \in I$. Finally, $I_{\gamma}^{2}\left(Z_{I}, Q_{I}\right)$ is wk $^{*}$ dense in $I_{\gamma}\left(E_{I}, Q_{I}\right)$ by Proposition 6.6 , so the result follows.

\section{References}

[1] C. Bennett and J. E. Gilbert, Homogeneous algebras on the circle. I. Ideals of analytic functions, Ann. Inst. Fourier (Grenoble) 22 (1972), no. 3, 1-19.

[2] L. Carleson, Sets of uniqueness for functions regular in the unit circle, Acta Math. 87 (1952), 325-345.

[3] P. L. Duren, Theory of $H^{p}$ Spaces, Academic Press, San Diego, 1970.

[4] K. M. Dyakonov, Equivalent norms on Lipschitz-type spaces of holomorphic functions, Acta Math. 178 (1997), 143-167.

[5] H. Hedenmalm, Bounded analytic functions and closed ideals, J. Anal. Math. 48 (1987), 142-166.

[6] K. Hoffman, Banach Spaces of Analytic Functions, Prentice-Hall, Englewood Cliffs, NJ, 1962.

[7] J. A. Johnson, Banach spaces of Lipschitz functions and vector-valued Lipschitz functions, Trans. Amer. Math. Soc. 148 (1970), 147-169.

[8] Y. Katznelson, An Introduction to Harmonic Analysis, Wiley, New York, 1968.

[9] B. I. Korenblum, Functions holomorphic in a disc and smooth in its closure, Dokl. Akad. Nauk SSSR 200 (1971), 24-27 (in Russian); English transl.: Soviet Math. Dokl. 12 (1971), 1312-1315.

[10] - Closed ideals in the ring $A^{n}$, Funktsional. Anal. i Prilozhen. 6 (1972), no. 3, 38-52 (in Russian); English transl.: Funct. Anal. Appl. 6 (1972), 203-214.

[11] A. L. Matheson, Closed ideals in rings of analytic functions satisfying a Lipschitz condition, in: Banach Spaces of Analytic Functions (Kent, OH, 1976), J. Baker et al. (eds.), Lecture Notes in Math. 604, Springer, Berlin, 1977, 67-72.

[12] - Approximation of analytic functions satisfying a Lipschitz condition, Michigan Math. J. 25 (1978), 289-298.

[13] - , Cyclic vectors for invariant subspaces in some classes of analytic functions, Illinois J. Math. 36 (1992), 136-144.

[14] D. R. Sherbert, The structure of ideals and point derivations in Banach algebras of Lipschitz functions, Trans. Amer. Math. Soc. 111 (1964), 240-272. 
[15] N. A. Shirokov, Division and multiplication by inner functions in spaces of analytic functions smooth up to the boundary, in: Complex Analysis and Spectral Theory (Leningrad, 1979/80), V. P. Khavin and N. K. Nikol'skiǔ (eds.), Lecture Notes in Math. 864, Springer, Berlin, 1981, 413-439.

[16] B. A. Taylor and D. L. Williams, Ideals in rings of analytic functions with smooth boundary values, Canad. J. Math. 22 (1970), 1266-1283.

[17] A. Zygmund, Trigonometric Series, vol. 1, Cambridge Univ. Press, 2nd ed., 1959.

Department of Mathematics and Physics

The Royal Veterinary and Agricultural University

Thorvaldsensvej 40

DK-1871 Frederiksberg C, Denmark

E-mail: vils@dina.kvl.dk

Received May 13, 2002

Revised version March 31, 2003 